ARTICLE

Received 14 Mar 2014 | Accepted 9 Dec 2014 | Published 5 Feb 2015 DOl: 10.1038/ncomms7053

\title{
MnSOD upregulation sustains the Warburg effect via mitochondrial ROS and AMPK-dependent signalling in cancer
}

Peter C. Hart ${ }^{1,2, \star}$, Mao Mao ${ }^{1,3, \star}$, Andre Luelsdorf P. de Abreu ${ }^{1,3,4, \star}$, Kristine Ansenberger-Fricano ${ }^{1,3}$, Dede N. Ekoue ${ }^{2}$, Douglas Ganini ${ }^{5}$, Andre Kajdacsy-Balla ${ }^{2}$, Alan M. Diamond ${ }^{2}$, Richard D. Minshall ${ }^{3,6}$, Marcia E.L. Consolaro ${ }^{4}$, Janine H. Santos ${ }^{7} \&$ Marcelo G. Bonini ${ }^{1,2,3}$

Manganese superoxide dismutase (MnSOD/SOD2) is a mitochondria-resident enzyme that governs the types of reactive oxygen species egressing from the organelle to affect cellular signalling. Here we demonstrate that MnSOD upregulation in cancer cells establishes a steady flow of $\mathrm{H}_{2} \mathrm{O}_{2}$ originating from mitochondria that sustains AMP-activated kinase (AMPK) activation and the metabolic shift to glycolysis. Restricting MnSOD expression or inhibiting AMPK suppresses the metabolic switch and dampens the viability of transformed cells indicating that the MnSOD/AMPK axis is critical to support cancer cell bioenergetics. Recapitulating in vitro findings, clinical and epidemiologic analyses of MnSOD expression and AMPK activation indicated that the MnSOD/AMPK pathway is most active in advanced stage and aggressive breast cancer subtypes. Taken together, our results indicate that MnSOD serves as a biomarker of cancer progression and acts as critical regulator of tumour cell metabolism.

\footnotetext{
${ }^{1}$ Department of Medicine, University of Illinois at Chicago, 909 South Wolcott Avenue, COMRB 1131, Chicago, Illinois 60612, USA. ${ }^{2}$ Department of Pathology, University of Illinois at Chicago, 909 South Wolcott Avenue, COMRB 1131, Chicago, Illinois 60612, USA. ${ }^{3}$ Department of Pharmacology, University of Illinois at Chicago, 909 South Wolcott Avenue, COMRB 1131, Chicago, Illinois 60612, USA. ${ }^{4}$ Universidade Estadual de Maringa, Avenida Colombo, 5790, CEP, 87020-900 Maringa, PR, Brazil. ${ }^{5}$ Free Radical Metabolite Section, Laboratory of Toxicology and Pharmacology, National Institute of Environmental Health Sciences/NIH, 111T.W., Alexander Drive MD-F02, Research Triangle Park, North Carolina 27709, USA. ${ }^{6}$ Department of Anesthesiology, University of Illinois at Chicago, 909 South Wolcott Avenue, COMRB 1131, Chicago, Illinois 60612, USA. ${ }^{7}$ Department of Physiology and Pharmacology, University of Medicine and Dentistry of New Jersey, 185 South Orange Avenue, Newark, New Jersey 07103, USA. * These authors contributed equally to this work. Correspondence and requests for materials should be addressed to M.G.B. (email: mbonini@uic.edu).
} 
A dvances in the understanding of the molecular basis of cancer have placed mitochondria ${ }^{1}$ at the centre of numerous abnormalities observed in tumour cell metabolism $^{2-4}$, differentiation ${ }^{5}$, proliferation $^{6}$ and survival ${ }^{7,8}$. Either because of its direct impact on the cellular metabolism or its role as a hub for signal transduction, deregulation of intrinsic mitochondrial processes combined with failure to halt cell cycle progression results in the genesis and progression of tumours $^{9-13}$. Among the many abnormal features of cancerous cells, a form of metabolism reliant on aerobic glycolysis is remarkable ${ }^{4}$, as it enables cell survival in the near absence of oxygen and provides the necessary building blocks to support vigorous proliferation. Recently, a growing number of studies aimed at defining mechanisms of mitochondrial deregulation in cancer have indicated that a deeper understanding of tumor cell metabolism will likely have an impact on therapeutics by enabling the development of targeted treatments with fewer complications and increased efficacy in preventing recurrence post therapy $7,14-18$

In parallel with glycolytic metabolism ${ }^{19-22}$, high MnSOD expression $^{23-25}$ is a distinctive feature of tumours particularly notable at advanced stages ${ }^{26,27}$. In healthy mitochondria, MnSOD directly regulates the metabolism of superoxide radical anions generated as a by-product of the electron transport chain. In isolation, MnSOD converts the diffusion-restricted, mild-oxidant superoxide radical into the diffusible, strong oxidant hydrogen peroxide $\left(\mathrm{H}_{2} \mathrm{O}_{2}\right)$ and thereby critically changes mitochondriadriven signalling in the cell. Thus, MnSOD does not always act as a first-line mitochondrial antioxidant defense. Recently, a study by our group demonstrated that in the absence of matched upregulation of mechanisms of $\mathrm{H}_{2} \mathrm{O}_{2}$ removal, MnSOD overexpression is actually detrimental to the integrity of mitochondria and the maintenance of its energetic functions ${ }^{28}$. This indicates that either directly or indirectly ${ }^{29,30}$ MnSOD regulates mitochondrial energetic and signalling functions. Using mitochondria-depleted cancer cells, it was established that the abrogation of mitochondria-dependent regulatory functions results in the appearance of highly invasive, aggressive, glycolytic cellular phenotypes ${ }^{31}$. Taken together, these observations indicate that progressive MnSOD upregulation, which results in mitochondrial dysfunction, could participate in the appearance of malignant cellular phenotypes characterized by glycolytic metabolism.

In this report, results are presented showing that mitochondrial MnSOD upregulation leads to the activation of AMP-activated kinase (AMPK), a cellular metabolic master switch ${ }^{32,33}$ that directly enhances glycolysis. We also establish that in cancer cells, $\mathrm{H}_{2} \mathrm{O}_{2}\left(\mathrm{mtH}_{2} \mathrm{O}_{2}\right)$ released from mitochondria consequentially to MnSOD upregulation is the signal that engages AMPK to produce and sustain the Warburg effect, thereby enabling cancer cell survival.

\section{Results}

MnSOD upregulated in cancer cells promotes glycolysis. In luminal breast cancer samples stratified by stage, MnSOD expression was present at significantly elevated levels in progressing tumour stages (Fig. 1a-d). The levels of MnSOD increased with histologic tumour grade being highest at histologic grade III and lowest in healthy and hyperplastic benign tissue (Fig. 1d). Elevated MnSOD levels were also observed in advanced prostate (Supplementary Fig. 1a) and colon (Supplementary Fig. 1b) cancer tissue as compared with healthy tissue samples. In breast cancer, MnSOD levels were noted to be highest in triplenegative and Her2 subtypes (Supplementary Fig. 2a), elevated in luminal cancers and lowest in healthy control tissue, indicating an a

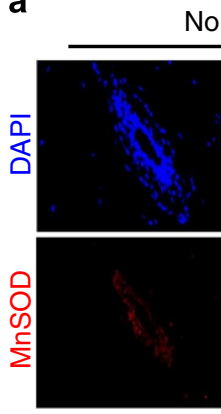

Normal
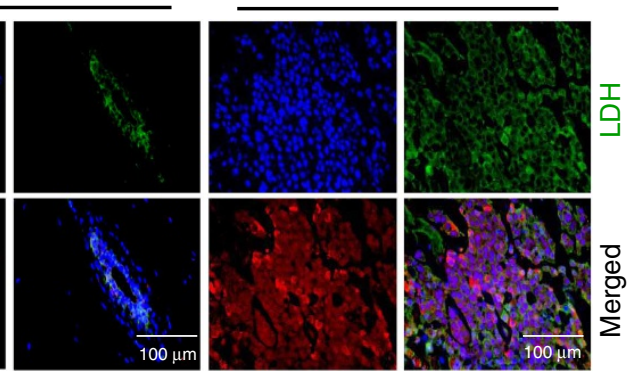

b

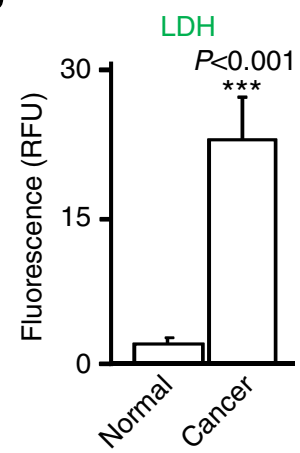

c

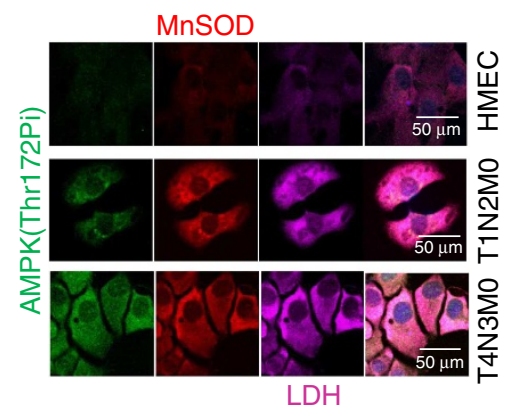

d

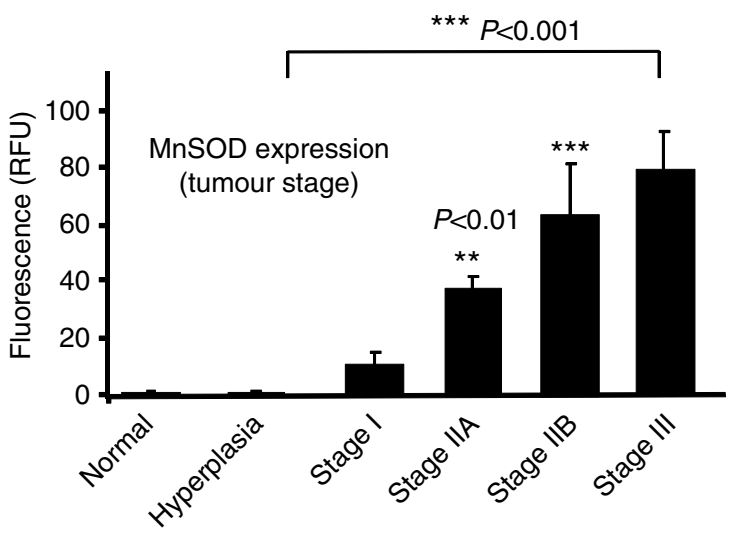

Figure 1 | Upregulation of MnSOD and the glycolysis surrogate LDH in human cancer tissue. (a) Representative immunostaining of MnSOD and $\mathrm{LDH}$ in normal versus cancer tissue. Normal tissue samples were obtained from breast reduction plastic surgery and represent examples of 'true healthy' tissue. Cancer tissue is a representative example of Stage III breast cancer collected, graded, processed and stored by the University of Illinois at Chicago tissue bank. Images are representative of over six independent cases of each kind. (b) Quantification of LDH expression in breast cancer (Stage III) as a surrogate of glycolytic metabolism. Quantification of corrected total fluorescence of LDH in all tissue samples was performed using ImageJ (imagej.nih.gov), and statistical analysis was performed using GraphPad InStat (GraphPad Software). (c) Immunostaining of MnSOD, LDH and phosphoAMPK (Thr172) in primary mammary epithelial cells (HMEC), cells derived from Her/Neu ( - ) p53 (+) ductal carcinoma (T1NOMO) and invasive ductal carcinoma Her/Neu( - ) p53(+) (T4N2MO). Analysis of MnSOD and LDH was performed using confocal microscopy. Images are representative of 15 different fields taken from three independent samples (five fields per dish). (d) Quantification of MnSOD in tissue microarrays containing normal and various cases of breast cancer at different pathologic stages (Protein Biotechnologies). Quantification of corrected total fluorescence of MnSOD was performed using ImageJ and statistical analysis was performed using one-way analysis of variance (ANOVA; GraphPad InStat). ${ }^{\star \star} P<0.01$;

$\star \star \star P<0.001$. For quantification of protein expression levels in patient samples (shown in $\mathbf{b}, \mathbf{d}$ ) a minimum of 15 independent cases were analysed. 
association between high MnSOD expression and tumour aggressiveness. This association was further strengthened by the epidemiologic analysis of published data $^{34}$ on 5 -year breast cancer survival, which negatively correlated with levels of MnSOD expression. Supplementary Fig. 2b shows the KaplanMeier distribution of 5-year breast cancer survival indicating a clear inverse relationship between survival and MnSOD levels. In all cancer types analysed (breast, prostate and colon), MnSOD expression was also correlated with expression of lactate dehydrogenase (LDH, Fig. 1b; Supplementary Fig. 1), a surrogate of glycolytic metabolism ${ }^{35,36}$ that is associated with poor prognosis. Quantification of total cell MnSOD and $\mathrm{LDH}$ fluorescence in breast (Fig. 1b), prostate and colon tissue (Supplementary Fig. 1c,d, respectively) indicated that these changes are significant and support a role for MnSOD upregulation in the switch to glycolysis, a common feature of aggressive cancer subtypes.

To assess whether MnSOD upregulation occurs during the progression towards malignancy, MCF10A (non-tumorigenic mammary epithelial cells) expressing oncogenic $\mathrm{v}$-Src kinase under the control of an inducible oestrogen receptor promoter were used. Activation of $\mathrm{v}$-Src expression by exposure of MCF10-Er-Src cells to tamoxifen resulted in MnSOD upregulation early during the process of malignant transformation (12 h, Supplementary Fig. 3) and progressively increased. Fully transformed cells $(48-72 \mathrm{~h})^{37,38}$ expressed the highest levels of MnSOD. The increase in MnSOD levels during transformation paralleled a strong metabolic shift towards glycolysis with notable reduction in the steady-state levels of ATP (Supplementary Fig. 3).

To begin to assess the impact of MnSOD upregulation on breast cancer cell metabolism, five isogenic cell lines stably overexpressing MnSOD were obtained. The control cell line expressing the empty vector (neo) and four additional clonal lines Mn1, Mn44, Mn11 and Mn28 expressing MnSOD at various levels (Fig. 2a) within the range observed in human breast cancer (Fig. 1d) were used to assess the impact of MnSOD expression on $\mathrm{O}_{2}$-dependent metabolism and glycolysis. In accordance with recent observations ${ }^{39}$, MnSOD activity increased with enhanced expression levels but not in a directly proportional manner (Fig. 2b). However, incremental increase in MnSOD dampened $\mathrm{O}_{2}$ uptake (Fig. 2c) in parallel to dissipating the mitochondrial electrochemical potential as assessed by JC-1 (Fig. 2d) and CMXROS (Fig. 2e). It was observed that when MnSOD levels were higher than five- to sixfold over basal (such as in the Mn44 clone) the electrochemical potential dropped in parallel with the reduction in the steady-state levels of ATP (Fig. 2f) and strong activation of compensatory glycolysis as assessed by lactate production (Fig. 2g). Elevated MnSOD expression levels were associated with progressive enhancement of $\mathrm{H}_{2} \mathrm{O}_{2}$ production (Fig. 2h), indicating a redirection in oxygen utilization from ATP production to $\mathrm{H}_{2} \mathrm{O}_{2}$ synthesis. Collectively, the findings summarized in Fig. 2 support the hypothesis that enhanced MnSOD expression sustains the metabolic switch in cancer cells. Further experiments using the $\mathrm{H}_{2} \mathrm{O}_{2}$ biosensor, roGFP targeted to mitochondria, indicated that $\mathrm{H}_{2} \mathrm{O}_{2}$ is locally generated in the organelle and that the increase in $\mathrm{H}_{2} \mathrm{O}_{2}$, as expected, paralleled the loss of mitochondrial electrochemical potential (Fig. 2h).

Using extracellular flow analysis (Seahorse Biosciences), we further examined the effect of MnSOD expression on mitochondrial respiration and the glycolytic rate. In agreement with the data shown in Fig. 2, cells expressing MnSOD at basal levels (neo) had significantly higher antimycin-inhibitable oxygen consumption rates (OCR) in comparison with Mn44 (moderate MnSOD expression) and Mn11 (high MnSOD expression Fig. 3a). Reserve respiratory capacity (the difference between maximal and basal respiratory rates) in all three cell lines was found to be significantly compromised indicating that in all cases mitochondria were functioning at the limit of their respiratory capacity even at baseline MnSOD activity in neo cells. Further confirmation of the increasing dependence of cells expressing high MnSOD levels on glycolysis was obtained in experiments where the extracellular acidification rate (ECAR) was measured (Fig. 3b). ECAR is a direct measure of lactic acid production by cells and thus of glycolytic activity. As shown in Fig. 3c, the OCR/ ECAR values for neo cells indicate that these cells are predominantly utilizing mitochondrial metabolism for energy. Higher levels of MnSOD expression in Mn44 and Mn11 promoted an increase in glycolysis (Fig. 3d). The relevance of this finding was highlighted by the observation that in the metastatic and heavily glycolytic breast cancer cell line (MDAMB-231), MnSOD expression levels were higher than those found in neo (MCF7 background; Fig. 4a). In comparison with MCF7/ neo, glycolytic and metastatic cells (MDA-MB231/U2OS) had MnSOD expression that matched those obtained in the Mn11 clone. Silencing MnSOD in MDA-MB231 cells (Fig. 4b) reduced the glycolytic rate of these cells with a parallel decrease in ATP steady-state levels (Fig. 4e,f) indicating that MnSOD-driven glycolysis significantly contributes to the maintenance of ATP production in phenotypically aggressive breast cancer cells.

MnSOD upregulation activates AMP-activated kinase. AMPK is a key energetic sensor and regulator of cellular metabolism that is activated by either a reduction in the intracellular ratio ATP/ AMP or by the elevation of intracellular $\mathrm{H}_{2} \mathrm{O}_{2}$ levels ${ }^{40}$. Because both a reduction in ATP and elevation of $\mathrm{H}_{2} \mathrm{O}_{2}$ were found to occur in parallel with MnSOD overexpression, we examined whether AMPK was activated in cells expressing incremental levels of MnSOD. Figure 5a shows that increasing levels of MnSOD expression increased AMPK activity as assessed both by the phosphorylation of active site Thr172 and the phosphorylation of the downstream target Acetyl CoA Carboxylase (ACC). Challenging the cells with exogenous $\mathrm{H}_{2} \mathrm{O}_{2}$, the product of MnSOD activity, significantly increased the phosphorylation and activity of AMPK in neo and Mn1 cells (Fig. 5a). Conversely, $\mathrm{H}_{2} \mathrm{O}_{2}$ only minimally increased AMPK phosphorylation and activity in Mn44 cells and did not further augment the phosphorylation or activity of AMPK in cells expressing high MnSOD levels (Mn11 and Mn28, Fig. 5a). These results indicated that the increase in MnSOD expression beyond levels found in Mn44 cells maximally activated AMPK by endogenously generating sufficiently high steady fluxes of $\mathrm{H}_{2} \mathrm{O}_{2}$ (Fig. 5a). We also confirmed that $\mathrm{mtH}_{2} \mathrm{O}_{2}$ was the molecular agent generated by $\mathrm{MnSOD}$ that led to AMPK activation. Neo cells were exposed to $(0-20 \mu \mathrm{M}) \mathrm{H}_{2} \mathrm{O}_{2}$, and AMPK phosphorylation, the phosphorylation of its downstream target ACC and the intracellular steady-state levels of ATP were assessed in side-by-side experiments (Supplementary Fig. 4). Low $\mathrm{H}_{2} \mathrm{O}_{2}$ levels insufficient to cause changes in ATP fully activated AMPK, indicating that $\mathrm{H}_{2} \mathrm{O}_{2}$ can induce AMPK activation independently of variations in ATP levels. To further demonstrate that when MnSOD activity is elevated $\mathrm{mtH}_{2} \mathrm{O}_{2}$ mediates the activation of AMPK, we expressed mitochondria-targeted catalase (mt-catalase) that efficiently dismutates $\mathrm{H}_{2} \mathrm{O}_{2}$ into $\mathrm{O}_{2}$ and $\mathrm{H}_{2} \mathrm{O}$ in mitochondria. Restricting $\mathrm{mtH}_{2} \mathrm{O}_{2}$ outflow resulted in a marked decrease in AMPK activation, especially in cells expressing higher levels of MnSOD (Fig. 5b). In accordance with our prediction that high MnSOD expression levels lead to a predominantly glycolytic metabolism (in order to maintain ATP production), mt-catalase did not change steady-state concentrations of ATP in neo cells but significantly reduced ATP levels by an additional $\sim 75 \%$ in 
a
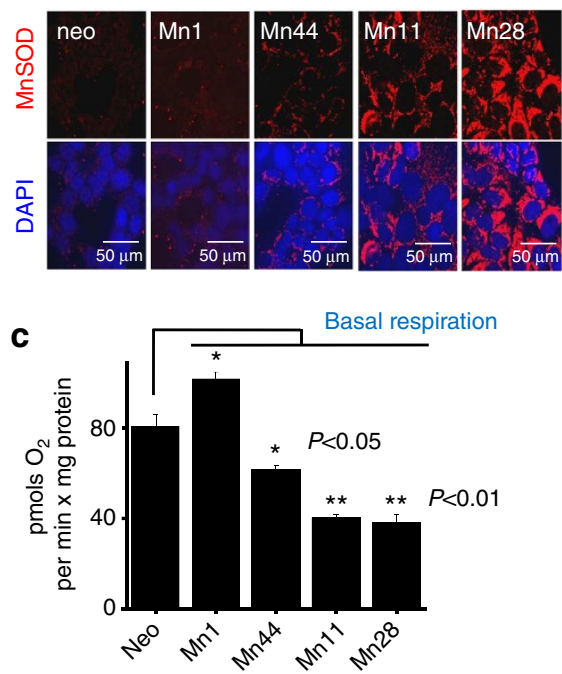

e
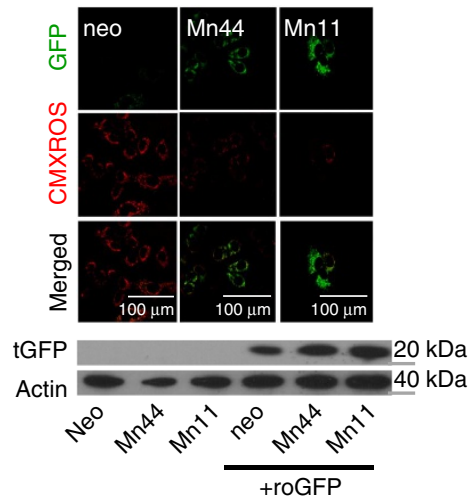

g

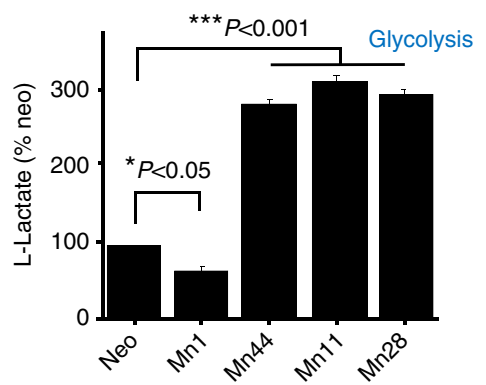

b

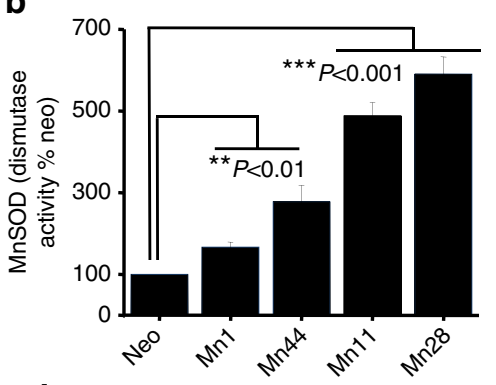

d

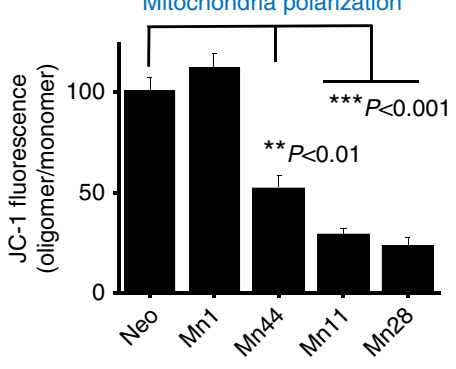

f

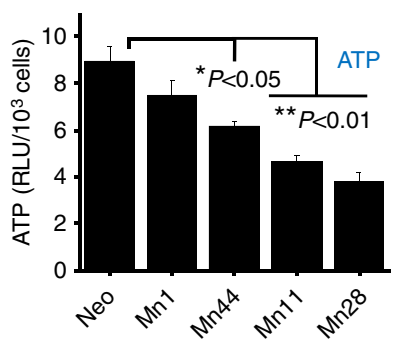

h

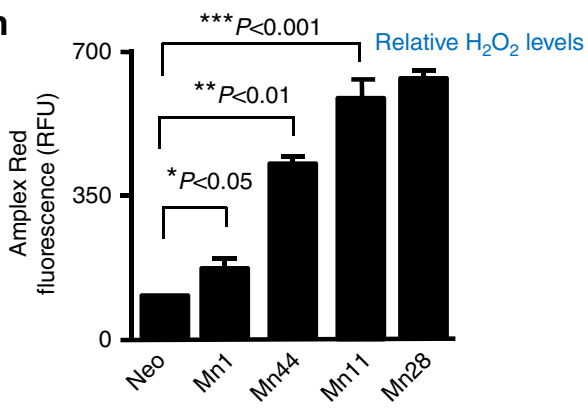

Figure 2 | MnSOD overexpression decreases mitochondrial metabolism and increases mitochondrial $\mathbf{H}_{\mathbf{2}} \mathbf{O}_{\mathbf{2}}$ outflow. (a) MnSOD-overexpressing epithelial breast cancer lines established in the MCF7 background were analysed by confocal microscopy for MnSOD expression levels. Representative images of 15 different fields were taken from three independent samples (five fields per dish). (b) Superoxide dismutase activity measured in immunoprecipitated-MnSOD from neo and the MnSOD-overexpressing cells. Activity was measured in competition assays with cytochrome c-Fe(III) and xanthine oxidase/hypoxanthine as the source of superoxide $(\mathrm{N}=3) .(\mathbf{c}) \mathrm{O}_{2}$ uptake measured by Seahorse in cells expressing incremental levels of MnSOD. MnSOD upregulation resulted in decreased basal respiration. Experiments were performed in triplicate. (d) Steady-state levels of ATP (measured with luciferin/luciferase) in cells expressing incremental levels of MnSOD. (e) Determination of the population of polarized/depolarized mitochondria using JC-1. For the analysis of polarized/depolarized mitochondria, confocal microscopy of live cells incubated with JC-1 (5 $\mu \mathrm{M}, 20$ min). Excess JC-1 was removed by washing the cells with PBS before analysis. The confocal microscope was set to read red and green channels simultaneously to avoid laser-induced photobleaching. (f) MnSOD overexpression resulted in increased glycolysis (assessed by lactate production rate) using the glycolysis cell-based assay kit (Cayman Chemical) according to the manufacturer's instructions. Cells were counted in the end of the experiments. Lactate production was normalized to cell number. (g) Overexpression of MnSOD resulted in increased output of $\mathrm{H}_{2} \mathrm{O}_{2}$, as measured by fluorescence from cells kept in culture using Amplex Red. Amplex Red was dissolved in DMSO and used at a final concentration of $5 \mu \mathrm{M}$ in phenol red-free media containing horseradish peroxidase $\left(0.1 \mathrm{U} \mathrm{ml}^{-1}\right)$. (h) Cells transfected with roGFP were treated with CMX-ROS $(1 \mu \mathrm{M})$, an indicator of mitochondrial potential in phenol red-free media, CMXROS accumulation in mitochondria was simultaneously assessed together with roGFP fluorescence by confocal microscopy of live cells. CMXROS was incubated with cells for $5 \mathrm{~min}$ before analysis. Overexpression of MnSOD correlated with an increase in oxidized roGFP (green) and a decrease in mitochondrial potential (red). Quantification of roGFP transfection efficiency by western blot is shown in $\mathbf{h}$. For all the experiments above, $n=6$ unless otherwise noted, ${ }^{\star} P<0.05 ;{ }^{\star \star} P<0.01 ;{ }^{\star \star \star} P<0.005$. Statistical analyses were performed using one-way ANOVA (GraphPad InStat). 
a
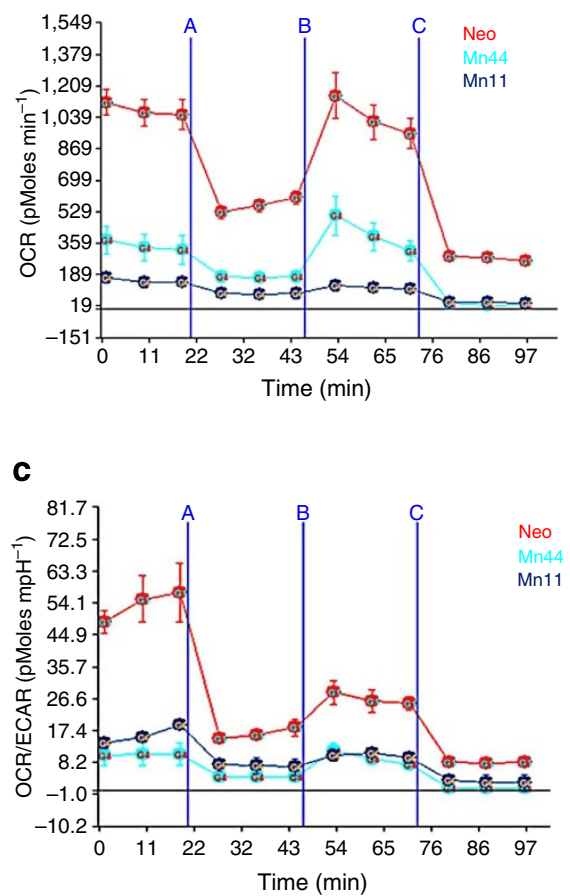

b

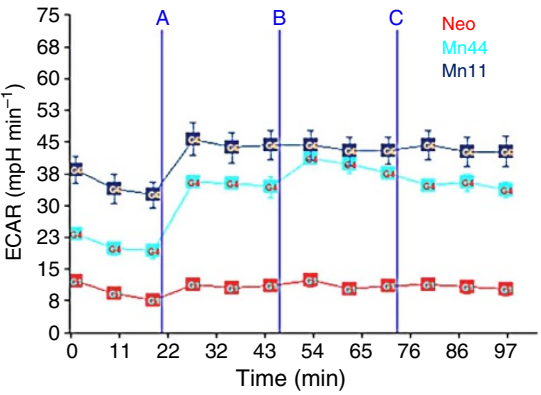

d

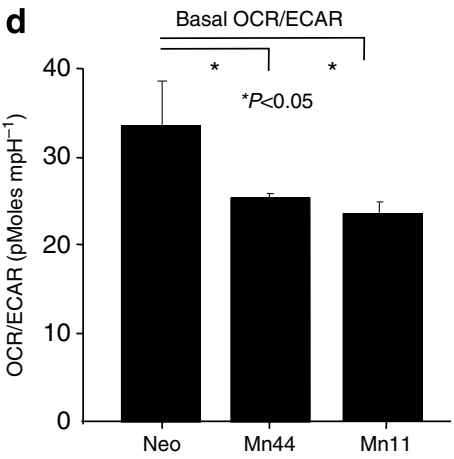

Figure 3 | MnSOD overexpression induces the glycolytic switch in MCF-7 breast cancer cells. Alterations in cellular metabolism were examined using Seahorse Bioscience XF Analyzer. Cells were plated as a monolayer in the Seahorse plate and left undisturbed until confluent. (a) OCR, an indication of OXPHOS and (b) ECAR, an indication of glycolysis, were measured. Mn 44 and Mn 11 cells expressing higher MnSOD levels had decreased OCR and increased ECAR compared with neo cells, (c) OCR/ECAR ratio was calculated and indicated that MnSOD overexpression produces cellular adaptation towards glycolytic metabolism. (d) Overall basal mitochondrial respiration was decreased in Mn44 and Mn11 versus neo as indicated by the ratio OCR/ECAR. Experiments are an average of three independent experiments. Statistical analysis was performed using one-way ANOVA with post hoc $t$-test (GraphPad InStat). ${ }^{\star} P<0.05(N=6)$.

Mn11 cells (Fig. 5c). Addition of mt-catalase also resulted in decreased glycolysis as assessed using lactate accumulation and the overall decrease in basal OCR/ECAR measured by extracellular flow analysis (Fig. 5d,e). Importantly, the increase in AMPK activity observed in the cells expressing high levels of MnSOD was also observed in breast, colon and prostate cancers (Fig. 5f,g). In addition, AMPK activation paralleled MnSOD upregulation during the course of MCF10-Er-Src transformation (Supplementary Fig. 3c), was enhanced in MDA-MB-231 in comparison with MCF7/neo (Fig. 4) and was suppressed following silencing of MnSOD in MDA-MB-231 (Fig. 4d). Together, these findings provide evidence for the involvement of MnSOD in promoting the glycolytic switch of aggressive breast cancer cells in an AMPK-dependent manner.

In view of recent reports implicating AMPK in the maintenance of NADPH levels in cancer cells, we also examined whether the MnSOD-directed activation of AMPK maintained steady-state NADPH levels in MnSOD-overexpressing cells (Supplementary Fig. 5). The levels of NADPH did not significantly change in cells overexpressing MnSOD when grown in complete media. However, in the absence of glucose, $\mathrm{NADPH}$ levels were diminished in MnSOD-overexpressing cells consistent with the high demand for NADPH in these cells that may have been sustained by the chronically elevated levels of $\mathrm{H}_{2} \mathrm{O}_{2}$ production. These results also indicate that $\mathrm{MnSOD}$, in a glucose-dependent manner, contributes to activate NADPH synthesis possibly via AMPK (Supplementary Fig. 5), thereby sustaining cancer cell biosynthetic capacity, viability and proliferation. AMPK phosphorylation and activity (as assessed by ACC phosphorylation) were found to parallel MnSOD expression in a tumour-stage-dependent manner (Supplementary Fig. 6).
AMPK activity was also found to be significantly increased in early malignant breast tumours as compared with control tissue and to increase progressively with tumour stage (Supplementary Fig. 6). In addition, in aggressive breast cancer subtypes, the elevation of MnSOD expression was strongly correlated with an increase in AMPK activation (Supplementary Fig. 6). This observation taken together with findings shown in Fig. 5f,g (that AMPK activation also parallels MnSOD upregulation in prostate and colon cancer) indicates the potential relevance of this finding as a mechanism to sustain aggressive cancer cell metabolism in multiple types of cancer.

MnSOD/AMPK direct the metabolic shift towards glycolysis. The activating effect of MnSOD upregulation on AMPK and the subsequent shift from cellular oxidative respiration to glycolysis was confirmed by silencing of MnSOD expression in MCF7/neo, Mn44 and Mn11 using short interfering RNA. Suppression of MnSOD expression in Mn44 and Mn11 was controlled to match levels of MnSOD expression equivalent to those found in resting MCF7/neo cells (Fig. 6a). AMPK phosphorylation analysed using western blot was observed to be reduced in Mn44 and in Mn11 cells transfected with MnSOD siRNA. Silencing of MnSOD caused significant suppression of glycolysis in Mn44 and Mn11 (Fig. 6b), while not significantly affecting MCF7/neo. Directly interfering with AMPK using a pharmacologic inhibitor (Compound C) significantly inhibited glycolysis in MCF7/neo, Mn44 and Mn11 cells as expected based on the prominent role of AMPK in activating glycolysis, but only affected ATP levels in Mn44 (mildly, -30\%) and Mn11 (heavily -85\%), Fig. 6d. Thus, while neo cells were capable of using different routes for 

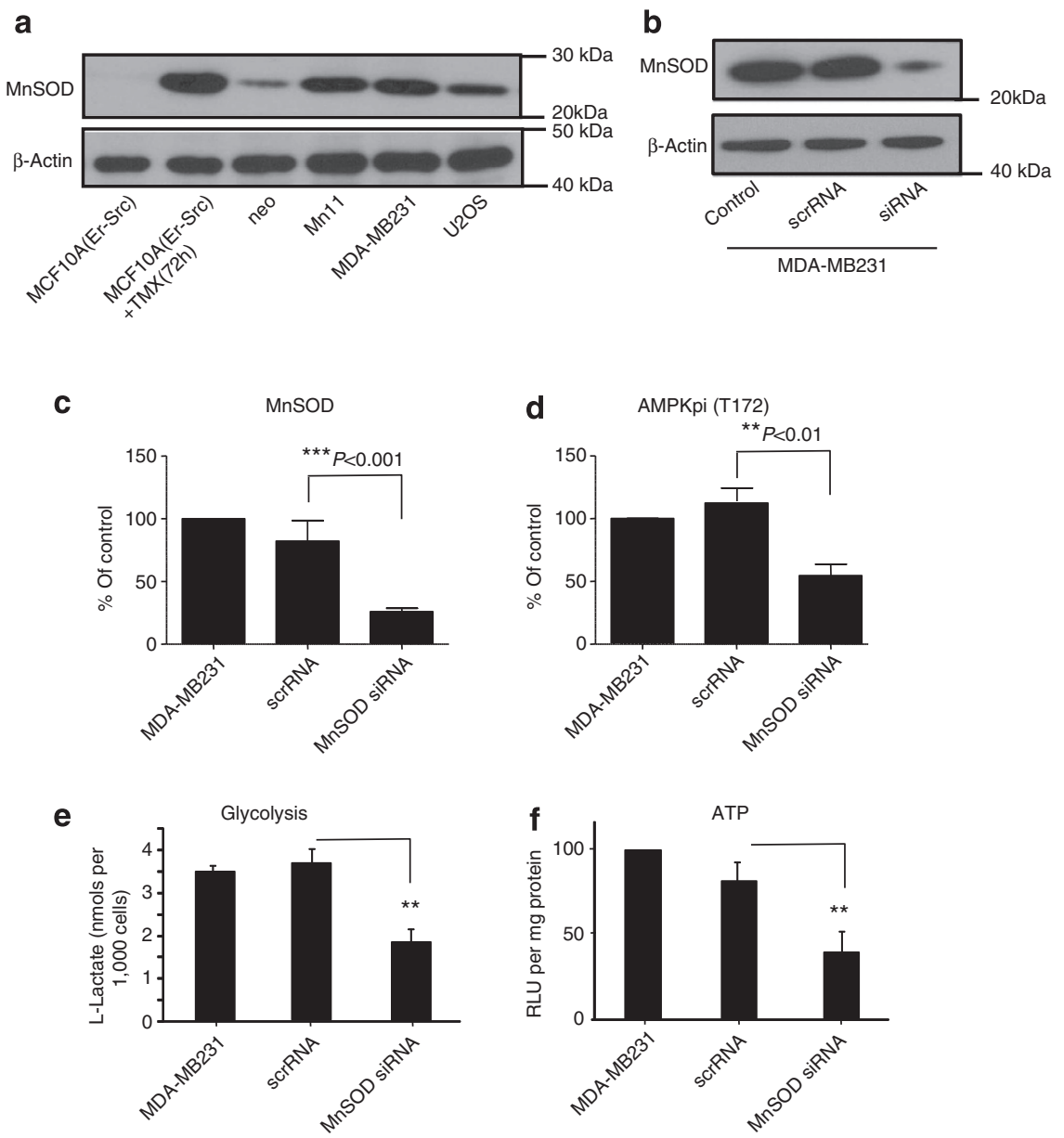

Figure 4 | Comparative MnSOD expression and effects in non-tumorigenic (MCF10A), tumorigenic (MCF7) and metastatic (MDA-MB-231 and U2OS) cells. (a) Western blot analysis of MnSOD expression levels in non-tumorigenic (non-induced MCF10AErSrc), tumorigenic (TMX-treated MCF10A-Er-Src, MCF7) and metastatic (MDA-MB-231 and U2OS) cell lines. (b) Levels of MnSOD in MDA-MB231 cells manipulated with MnSOD-targeted siRNA and respective diminishment in AMPK phosphorylation levels as analysed by western blot. (c) Quantification of MnSOD expression by western blot before and after treatment of MDA-MB231 cells with silencing RNA. (d) Quantification of AMPK phosphorylation before and after MnSOD silencing in MDA-MB231. (e) Effect of MnSOD silencing on the glycolytic rate of MDA-MB-231 cells measured by extracellular flow analysis using Seahorse Biosciences XF analyser. (f) Effect of MnSOD silencing on steady-state ATP levels of MDA-MB-231 cells. Cells were harvested and analysis was performed $72 \mathrm{~h}$ after the delivery of siRNA. Statistical analysis was performed using one-way ANOVA with post hoc $t$-test (GraphPad InStat) ${ }^{\star} P<0.05 ;{ }^{\star \star} P<0.01$; ${ }^{\star \star \star} P<0.001$; for $\mathbf{b}$-e statistical analysis was performed using at least four independent replicates. Quantification and statistical analysis of $G$ used at least 15 cases classified according to clinical stage and histologic grade.

ATP synthesis, cells expressing higher levels of MnSOD appeared to have increasing dependence on glycolysis. This finding was further examined in cells devoid of AMPK's catalytic subunit $A M P K \alpha 1$. To investigate the effect of MnSOD/AMPK in the induction of the Warburg effect, MnSOD was transfected into MCF-7 cells constitutively expressing AMPK $\alpha 1$ siRNA versus scrRNA. Results shown in Fig. 6e,f show that MnSOD overexpression in AMPK-competent cells recapitulated the strong repression of $\mathrm{O}_{2}$ uptake observed in $\mathrm{Mn} 11$ cells in parallel with an increase in the ECAR (an index of increased glycolytic metabolism). Conversely, overexpression of MnSOD in cells devoid of AMPK $\alpha 1$ produced only marginal effects on cellular metabolism that remained predominantly aerobic.

The dependence of MnSOD-overexpressing cells on glucose was also exemplified by the finding that Mn44 and Mn11 could only maintain steady levels of NADPH in a glucose-rich medium (Supplementary Fig. 5) consistent with the expectation that higher intracellular $\mathrm{H}_{2} \mathrm{O}_{2}$ leads to accelerated NADPH turnover. These observations are in agreement with recent published work demonstrating that one of the many important functions of
AMPK activation in cancer is to maintain NADPH recycling and availability $^{41}$ (Supplementary Fig. 5).

MnSOD overexpression upregulates glycolytic enzymes. Additional insight into the importance of the MnSOD/AMPK pathway for the glycolytic shift of cancer cells was obtained by determining whether MnSOD levels affected the expression and activity of the key glycolytic enzymes. Hexokinases are enzymes that catalyse glucose phosphorylation for entry in the glycolytic and pentose phosphate pathways, and phosphofructokinases (PFK) catalyse the rate-limiting step in the glycolytic pathway. Hexokinase and PFK activities were found to be enhanced in cells expressing high MnSOD levels in comparison with neo controls. PFK activity was increased consistent with a greater reliance of MnSOD-overexpressing cells on glycolysis (Supplementary Fig. 7) and the direct activating effect of AMPK on PFK2. In fact, it was confirmed that MnSOD overexpression in AMPK-competent cells resulted in remarkable phosphorylation of PFK2 on Ser466, an activating AMPK-dependent phosphorylation site ${ }^{42}$ 
a

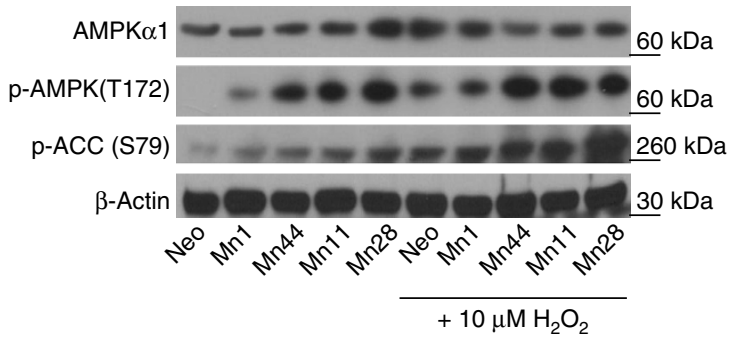

c

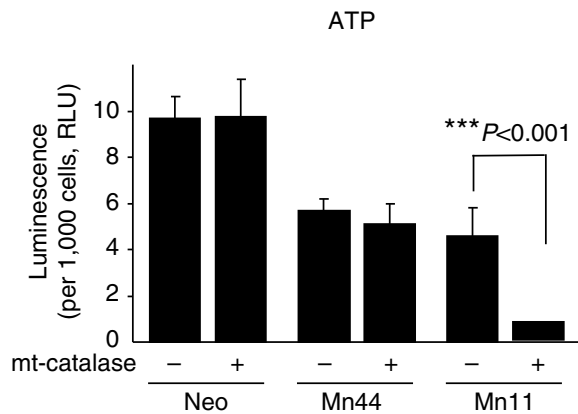

e

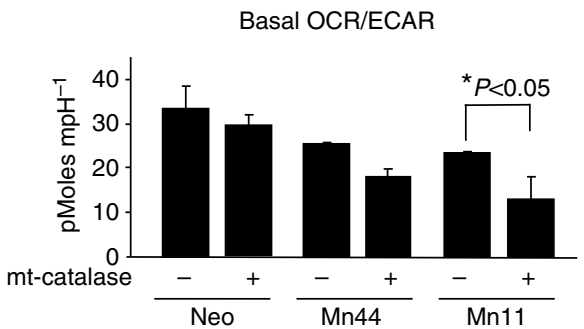

g

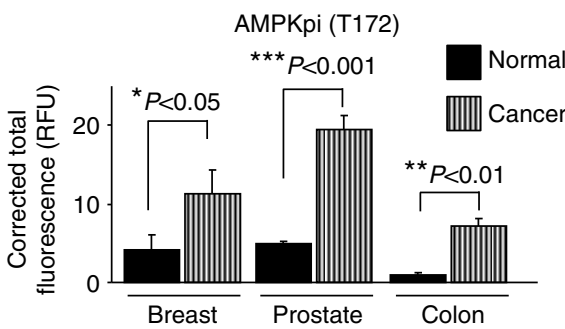

b

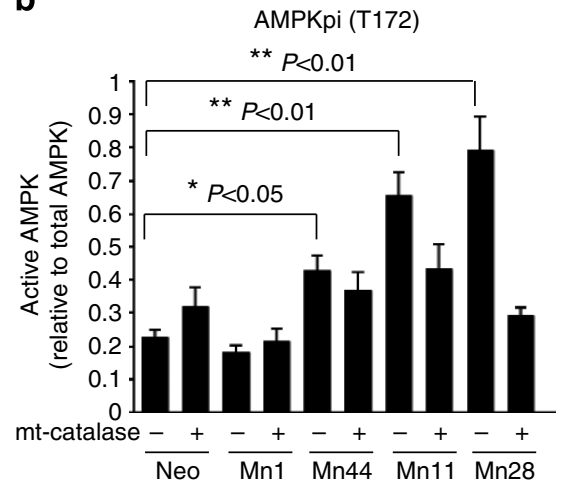

d

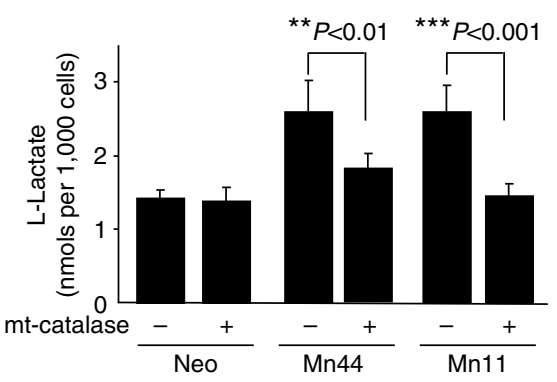

f
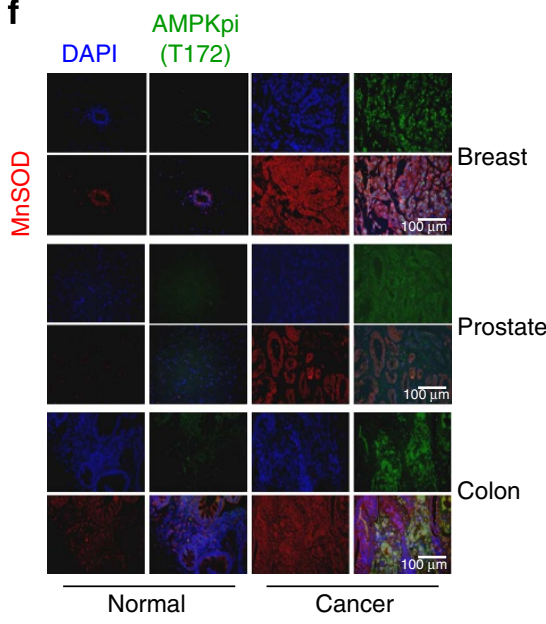

Figure 5 | AMPK activation is required for the MnSOD-driven glycolytic shift. (a) Western blot analysis of AMPK activation. Active AMPK was examined using phospho-AMPK $\alpha 1$ (Thr172) and phospho-ACC (Ser 79) as surrogates. Treatment of cells with exogenous $\mathrm{H}_{2} \mathrm{O}_{2}(10 \mu M)$ for 30 min significantly increased AMPK activation in neo, Mn1 and Mn44 but did not further enhanced AMPK activity in Mn11 and Mn28. (b) Transfection of mitocatalase into neo and MnSOD-overexpressing cells reduced AMPK activation in Mn11 and Mn28 indicating that $\mathrm{H}_{2} \mathrm{O}_{2}$ originating from mitochondria activates AMPK. The Mitocatalase gene was delivered to MCF7 cells using a lentiviral construct $72 \mathrm{~h}$ before harvesting. (c) Treatment with mitocatalase reduced steady-state levels of ATP in Mn11 indicating that cells expressing high MnSOD levels depend on mt $\mathrm{H}_{2} \mathrm{O}_{2}$ and AMPK activation to support ATP production. Steady-state ATP levels were assessed by a chemiluminescence employing luciferin/luciferase mixtures. (d) Diminished lactate production by mitocatalase in $\mathrm{Mn} 44$ and $\mathrm{Mn} 11$ further indicated that $\mathrm{MnSOD}$ overexpression via $\mathrm{mtH}_{2} \mathrm{O}_{2}$ enhances glycolysis, which in turn sustains ATP production. (e) Mitocatalase did not affect the OCR/ECAR ratio in neo cells but significantly altered OCR/ECAR in Mn44 and Mn11 indicating that MnSOD overexpression leads to the metabolic shift to glycolysis via increasing $\mathrm{mtH}_{2} \mathrm{O}_{2}$ outflow. (f) Staining of phospho-AMPK $\alpha 1$ (Thr172) in cancerous human breast, prostate and colon tissue compared with control normal tissue. AMPK phosphorylation (green staining) paralleled an increase in MnSOD expression (red staining). Tissues were collected, processed, graded and stored by the University of Illinois tissue bank. (g) Quantification of phospho-AMPK $\alpha 1$ (Thr172) and MnSOD fluorescence staining performed using ImageJ, and statistical analysis was performed by one-way ANOVA (GraphPad InStat). ${ }^{\star} P<0.05$; ${ }^{\star \star} P<0.01$; $\star \star \star P<0.001$.

(Supplementary Fig. 7b). MnSOD overexpression in AMPKdeficient cells did not result in increased PFK2 phosphorylation supporting our hypothesis that MnSOD sustains the glycolytic activity of cells where it is overexpressed by engaging AMPKdependent signalling (Supplementary Fig. 7). Notably, a marked reduction in the expression levels of mitochondrial electron 
a

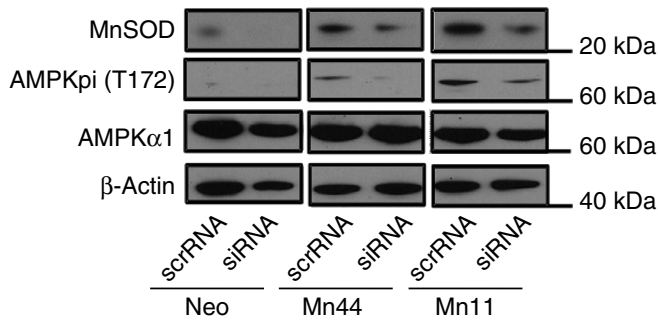

b

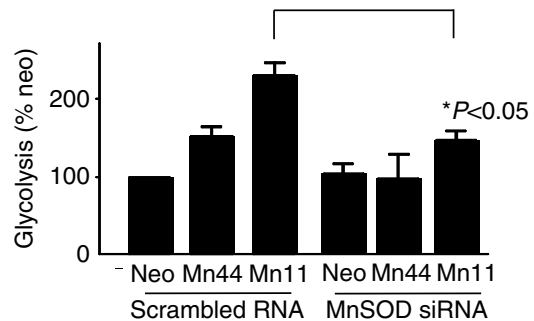

C

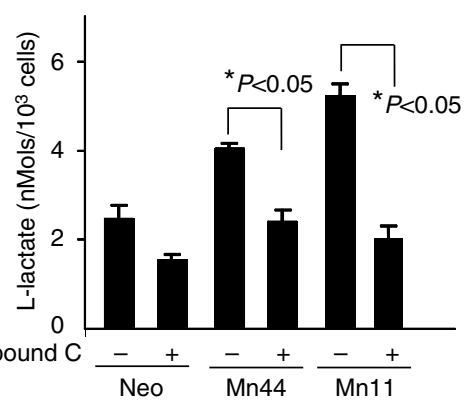

e

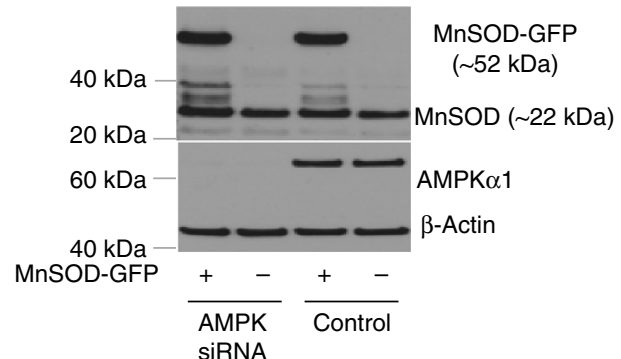

d

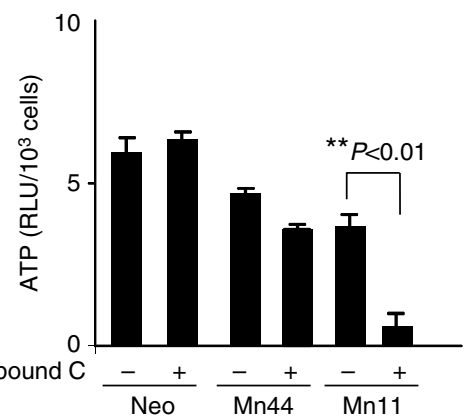

$\mathbf{f}$

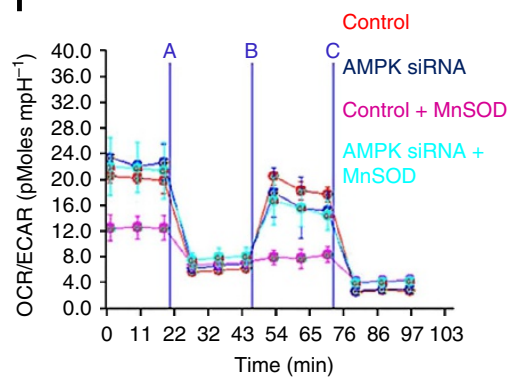

Figure 6 | Suppression of MnSOD or inhibition of AMPK impair the shift to glycolysis in cells overexpressing MnSOD. MnSOD siRNA was introduced into cells overexpressing MnSOD by electroporation. Cells were harvested $48 \mathrm{~h}$ after siRNA delivery. (a) Western blot of protein lysates showed the decrease of MnSOD expression in Mn44 and Mn11 to levels compared with undisturbed neo. The partial downregulation of MnSOD in Mn44 and Mn11 corresponded to a decrease in activated AMPK (Thr172p) analysed by western blot. (b) MnSOD siRNA transfection resulted in decreased glycolytic rate compared with untreated cells. The glycolytic rate was assessed by monitoring lactate production between 48 and $52 \mathrm{~h}$ post-MnSOD silencing. (c) AMPK inhibition with compound C (for $24 \mathrm{~h}, 25 \mu \mathrm{M}$ ) decreased glycolysis. (d) ATP steady-state levels were slightly and markedly decreased in Mn44 and Mn11, respectively, by the inhibition of AMPK with compound C. (e) Cells constitutively expressing AMPK $\alpha 1$ siRNA were transfected with MnSOD-GFP or GFP carrying constructs using lipofectamine. Western blot analysis of MnSOD expression levels was performed by western blot $48 \mathrm{~h}$ after transfection. (f) Cells overexpressing MnSOD on an AMPK-competent and AMPK-depleted background were analysed by extracellular flow analysis (seahorse). MnSOD overexpression led to the metabolic shift to glycolysis only when expressed in AMPK-competent cells. ${ }^{\star} P<0.05 ;{ }^{\star \star} P<0.01 ; N=3$ independent replicates.

transfer chain complexes II, III, IV and V (Supplementary Fig. 8a) was observed in cells overexpressing MnSOD consistent with the observations of reduced mitochondrial potential and $\mathrm{O}_{2}$ uptake. In parallel, we analysed the expression of the key antioxidant enzymes in order to gain additional understanding of the mechanisms underlying the persistent elevation in $\mathrm{H}_{2} \mathrm{O}_{2}$ due to $\mathrm{MnSOD}$ upregulation. Catalase, glutathione reductase, thioredoxin and thioredoxin reductase were examined and only thioredoxin was significantly reduced in Mn44, Mn11 and Mn28 cells (Supplementary Fig. 8b). These results indicate that alterations in the expression and functioning of electron transfer chain complexes in MnSOD-overexpressing cells enhance $\mathrm{mtH}_{2} \mathrm{O}_{2}$ production unopposed by the major $\mathrm{H}_{2} \mathrm{O}_{2}$-scavenging systems.

CaMKII, a redox-sensitive kinase that activates AMPK. Data presented above indicated that the constitutive elevation of cellular $\mathrm{H}_{2} \mathrm{O}_{2}$ maintained AMPK activation thereby promoting the irreversible conversion of the cellular metabolism to glycolytic. The mechanism of MnSOD-dependent AMPK constitutive activation was next investigated with a focus on CaMKII, a kinase responsive to $\mathrm{H}_{2} \mathrm{O}_{2}$-driven oxidative activation upstream of $\mathrm{AMPK}^{43,44}$. Oxidation of CaMKII at residues M281 and M282 had previously been shown to result in chronic activation, and this constitutive activation of CaMKII was apparent even in cells overexpressing low levels of MnSOD (two- to threefold over basal, Mn1), Fig. 7a. Consistent with the hypothesis that AMPK is critical for maintaining increased glycolysis and steadystate ATP levels in MnSOD overexpressing cells, the CaMKII inhibitor KN93 dampened glycolysis and reduced the steady-state ATP levels in Mn44 and Mn11 cells (Fig. 7b). Importantly, inhibition of CaMKII resulted in robust dephosphorylation of AMPK at the active site Thr172 (Fig. 7c). Decreased AMPK phosphorylation was accompanied, as expected, by a reduction in AMPK activity as indicated by lower levels of phosphorylated ACC (Fig. 7c), a downstream target of AMPK. This observation indicates that CaMKII oxidation by $\mathrm{mtH}_{2} \mathrm{O}_{2}$ leads to its 

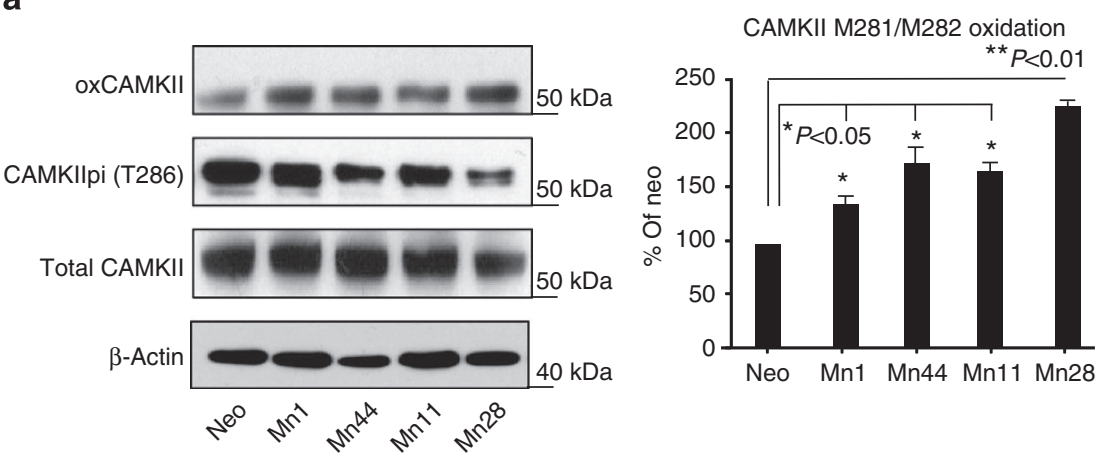

b

Percent change in glycolysis by CaMKII inhibitor KN93 (5 $\mu \mathrm{M}, 36 \mathrm{~h})$
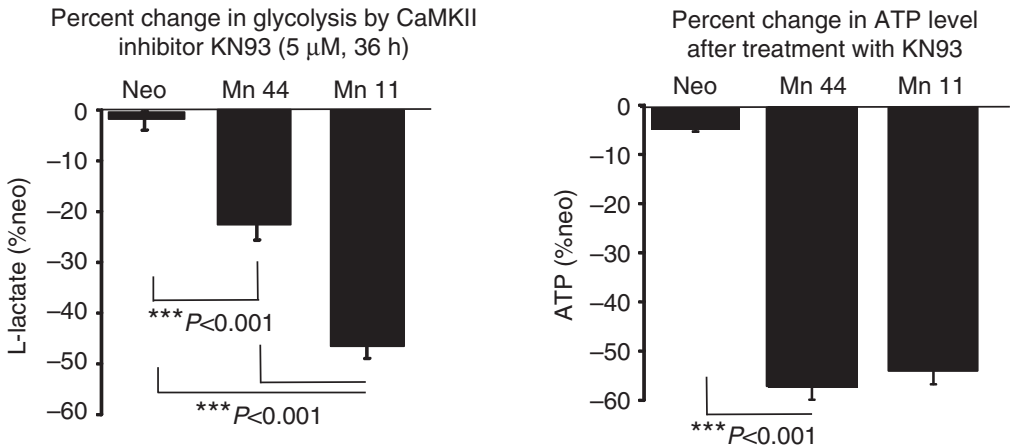

C

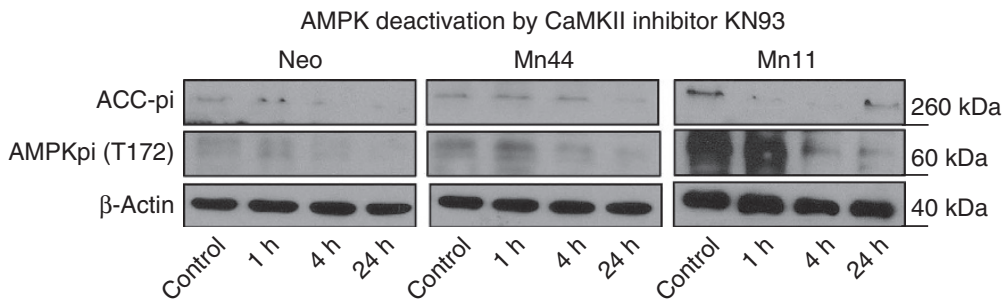

Figure 7 | Calmodulin kinase II (CaMKII) is a redox-sensitive kinase upstream of AMPK. (a) CaMKII oxidation was examined by western blot using an antibody recognizing the oxidation of M281/M282 to methionine sulfoxide on CaMKII. Panel to the right shows quantification by densitometry of oxidized CaMKII detected in three independent experiments. (b) Impact of KN93 (a bona fide CaMKII inhibitor) on lactate production and steady ATP levels in neo, Mn44, Mn11. Water-soluble KN93 ( $5 \mu \mathrm{M}$ ) was diluted into complete media. Cells were harvested at $36 \mathrm{~h}$ for assessments of lactate production and steady-state levels of ATP as described in methods. (c) The impact of CaMKII inhibition with KN93 on AMPK phosphorylation and activity (pACC was used as a surrogate) was examined. Cells exposed to KN93 $(5 \mu \mathrm{M})$ were harvested at the indicated time points after KN93 addition to full media, lysed and analysed for AMPK(Thr172p) and pACC (Ser79) content by western blot. ${ }^{\star} P<0.05 ;{ }^{\star \star} P<0.01 ;{ }^{\star \star \star} P<0.01$. Statistical analysis was performed using six independent replicates.

constitutive activation and consequentially the activation of AMPK. Inhibition of CaMKII markedly reduced the viability of cells expressing high MnSOD levels (Fig. 8). The potential clinical importance of this finding was furthered by comparing the effect of CaMKII inhibitor KN93 with two commonly used breast cancer chemotherapeutic compounds, paclitaxel (a mitotic inhibitor) and 5-fluorouracil (a thymidylate synthase inhibitor). Exposure to KN93 resulted in $60-70 \%$ cell death of Mn44 and Mn11 cells while only minimally reducing the viability of MCF7/ neo cells by $\sim 20 \%$, which was in clear contrast to the effect of paclitaxel and 5-flurouracil, which were far more efficient in killing MCF7/neo (Fig. 8). This observation indicated that cells expressing high MnSOD levels are far more sensitive to CaMKII inhibition than to paclitaxel or 5-fluorouracil.

Glycolysis maintains cells overexpressing MnSOD viable. The dependence of cells expressing high MnSOD levels on glycolysis was further examined by conducting experiments where glycolysis was inhibited using 2-deoxy-D-glucose (2-DG). MCF7/neo,
Mn44 and Mn11 cells were treated with 2-DG (a potent glucose analogue that cannot be metabolized) and then apoptosis was measured by flow cytometry (Fig. 9). 2-DG produced marked cell death among Mn11 cells compared with MCF7/neo controls. The inhibition of glycolysis dramatically lowered steady-state ATP levels in Mn44 and Mn11 cells, while only moderately inhibiting ATP production in neo cells (Fig. 9b). These results indicate that glycolysis significantly contributes to ATP production in MCF7/neo cells, but accounts for most, if not all, of the ATP production in cells expressing high MnSOD levels. Accordingly, we found that supplementation of media with substrates that support glycolytic metabolism (pyruvate and uridine) stimulated Mn11 and Mn28 proliferation without affecting MCF7/ neo or Mn1 growth rates (Supplementary Fig. 9). The proliferation rate of Mn11 and Mn28 in the presence of pyruvate and uridine was comparable to the baseline proliferation rate of MCF7/neo, dismissing the concept that MnSOD per se is a tumour suppressor. Rather, MnSOD imposes a form of metabolism that requires glycolysis-supportive substrates to maintain rapid cellular proliferation. 
Activation of the MnSOD/AMPK axis suppresses apoptosis. MnSOD is known to promote cell survival and inhibit apoptosis by mechanisms that remain poorly defined ${ }^{45}$. Since AMPK has been implicated in upregulating Bcl-2/survivin expression ${ }^{46}$, we examined the possibility that in addition to the metabolic effects described above, MnSOD/AMPK fostered cancer cell survival by

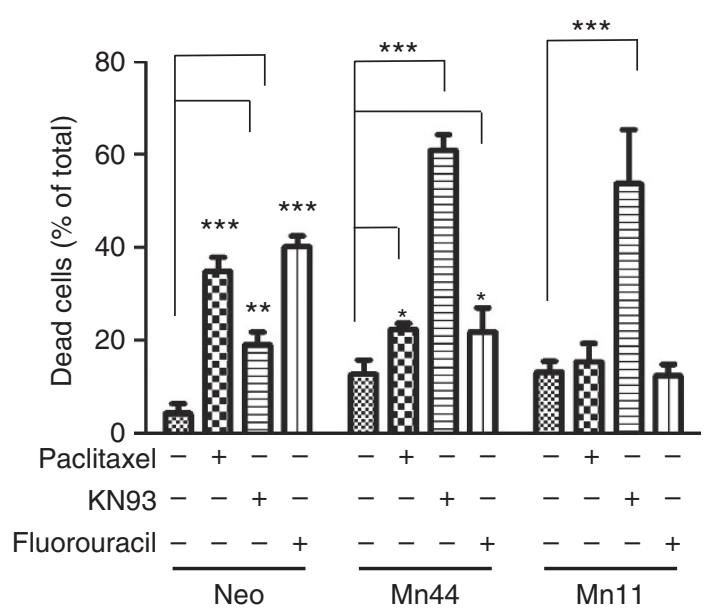

Figure 8 | Comparative effect of anticancer drugs paclitaxel $(5 \mu \mathrm{M})$, 5-fluorouracil ( $2 \mathrm{mM})$ and the CaMKII inhibitor KN93 (5 $\mu \mathrm{M})$ on cellular viability. Cells (neo, Mn44 and Mn11) were exposed to the specified compounds as indicated for $48 \mathrm{~h}$ in full media. After $48 \mathrm{~h}$ cells were harvested and viability was determined by flow cytometry using YOPRO and propidium iodide as described in Methods. Experiments show averages of six independent experiments. Statistical analysis was performed using one-way ANOVA (GraphPad InStat) ${ }^{\star} P<0.05$; ${ }^{\star \star} P<0.01$; ${ }^{\star \star \star} P<0.001$. upregulating anti-apoptotic factors such as survivin and Bcl-2. Cells expressing high MnSOD levels also had concurrent increases in survivin and Bcl-2 (Fig. 10a,b). To test the involvement of AMPK in the induction of survivin and Bcl-2 expression by MnSOD, AMPK expression was reduced using siRNA, which resulted in decreased Bcl-2 and survivin levels in both Mn44 and Mn11 cells. This observation implicates AMPK as a critical mediator of MnSOD-induced anti-apoptosis (Fig. 10c). Expression of MnSOD and survivin/Bcl-2 was also similarly increased in human cancer tissues compared with control tissue (Fig. 9d). Furthermore, KN93 was observed to be a potent inducer of apoptosis of MnSOD-overexpressing cells, likely because of additive effects in suppressing AMPK-driven metabolic and antiapoptotic routes (Fig. 8). These findings support a critical role for MnSOD/oxCaMKII/AMPK in the maintenance of glycolytic cancer cell survival while adding mechanistic insight to the known anticancer effect of CaMKII inhibition ${ }^{45,47}$. MnSOD upregulation also repressed the cleavage of effector caspases 3 and 7 while activating caspase 9 and downregulating p53 expression (Supplementary Fig. 10). These findings further substantiate the critical role of MnSOD in adaptative responses that favour cancer cell survival. Our studies also indicate that MnSOD upregulation produced phenotypes that metabolically and functionally resemble more malignant cells such as those that occur in latestage and aggressive subtypes of breast cancers. This conclusion was further supported by experiments using MCF10-Er-Src cells where MnSOD upregulation was shown to be essential for the survival of transformed cells (Supplementary Fig. 11). Silencing MnSOD before induction of MCF10A-Er-Src transformation induced by tamoxifen led to $>70 \%$ cell death $48 \mathrm{~h}$ after tamoxifen stimulation. Although MnSOD ectopic expression did not significantly increase metastasis of xenografts produced in NOD/SCID mice, enhanced MnSOD expression in breast cancer cells conferred characteristics of aggressive cancer cells such as a
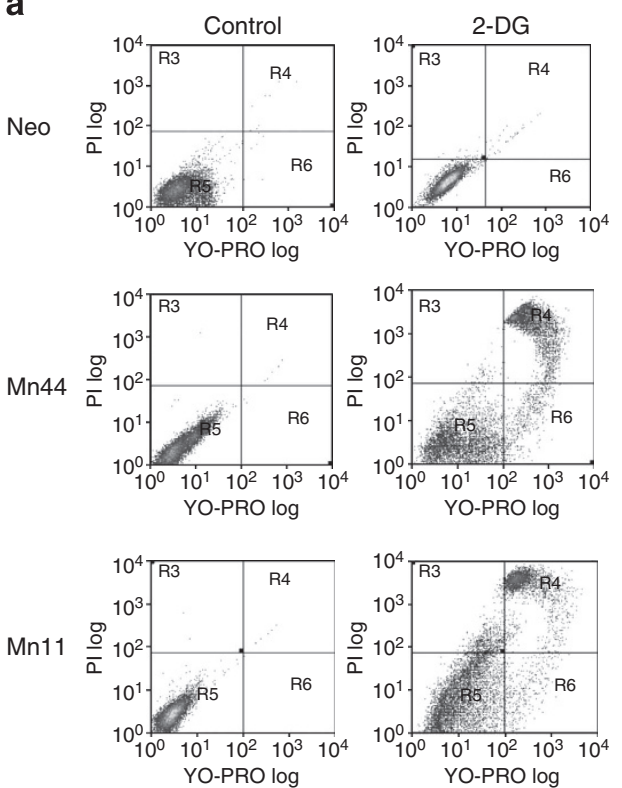

b

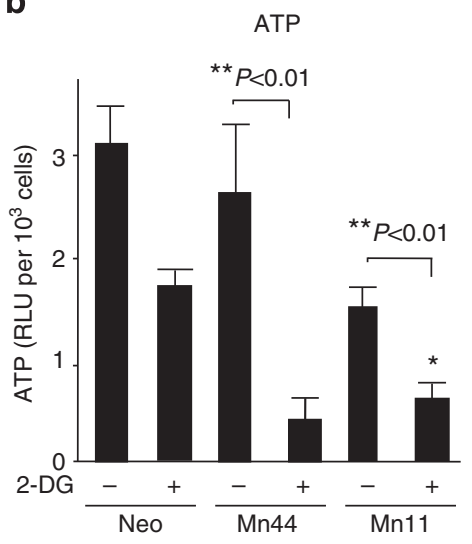

Figure 9 | Inhibition of glycolysis decreased viability and ATP steady-state levels in lines expressing high MnSOD levels. Cells were treated with the glucose competitor 2-DG (10 mM for $36 \mathrm{~h}$ ) and analysed by flow cytometry using YOPRO, an indicator of apoptosis and propidium iodide, an indicator of cell death. (a) Cells overexpressing MnSOD were more susceptible to 2-DG-induced apoptosis compared with control cells (neo). Mn44 and Mn11 cells as expected were the most vulnerable to glycolysis inhibition. (b) In cells treated with 2-DG, there were also decreases in steady-state ATP levels analysed by chemiluminescence $36 \mathrm{~h}$ after 2-DG addition to the media. Reductions in ATP levels were marked in Mn44 and Mn11 confirming the reliance of these cells on glycolysis for ATP production. Statistical analysis was performed using one-way ANOVA (GraphPad InStat) ${ }^{\star \star P}<0.01$; $N=3$ independent experiments. 
a

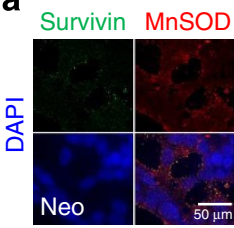

$\mathrm{BCl}-2 \mathrm{MnSOD}$
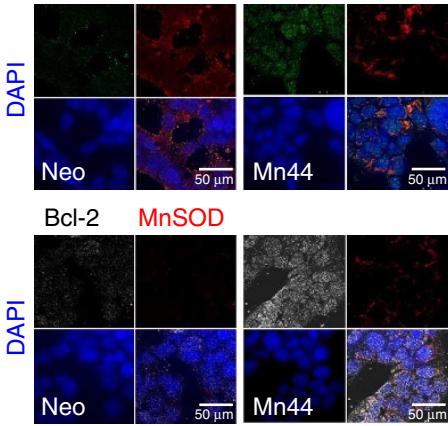

Mn44

Mn44

c
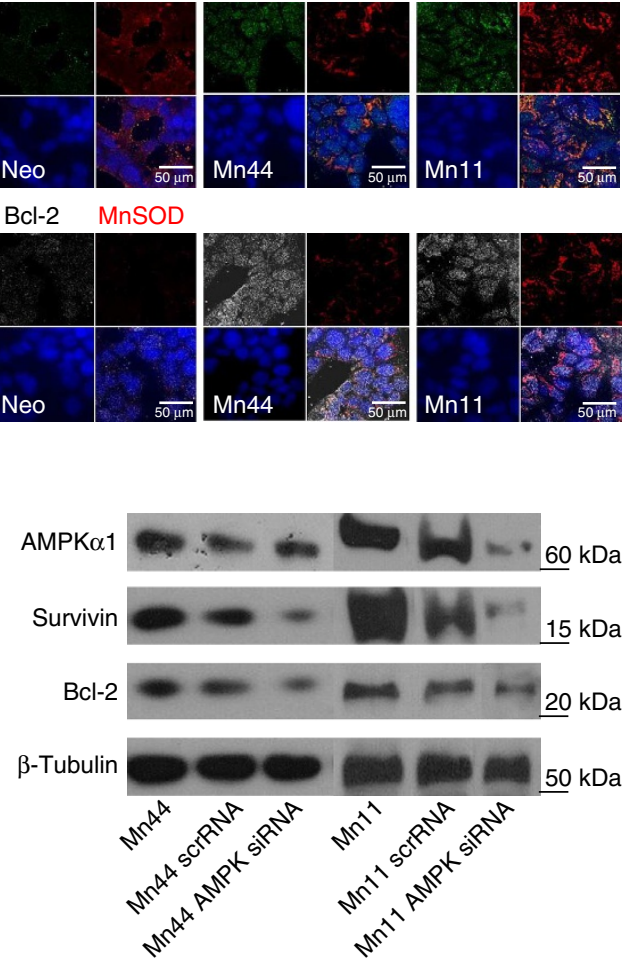

\section{b}

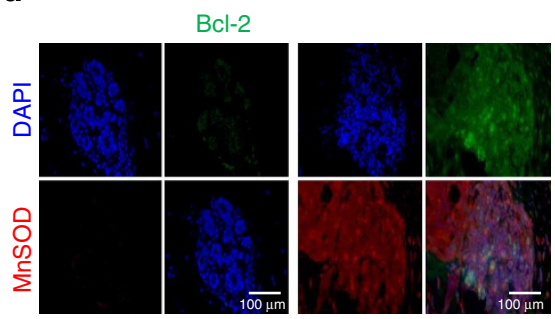

Survivin

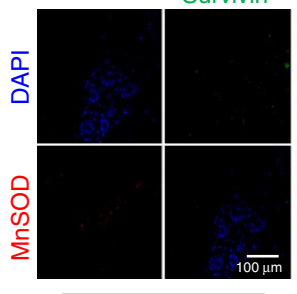

Normal breast

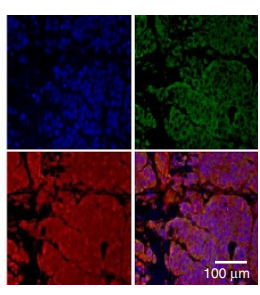

Cancer

Figure 10 | MnSOD induces the expression of survival proteins in an AMPK-dependent manner. (a) Survivin and Bcl-2 protein expression levels using protein extracts from neo, Mn44 and Mn11 cells were measured by confocal microscopy. Panel (b) same is as (a), but protein expression was analysed by western blot in neo, Mn1, Mn44, Mn11 and Mn28 cells. (c) Silencing of AMPK by siRNA transfection with lipofectamine decreases Bcl-2 and survivin expression in Mn44 and Mn11 (results from the analysis of Mn44 and Mn11 are from different sections of the same nitrocellulose membrane). AMPK siRNA was transfected with lipofectamine $72 \mathrm{~h}$ before harvesting. (d) Immunohistochemical analysis shows increased expression of anti-apoptotic proteins survivin and $\mathrm{Bcl}-2$ in human breast cancer tissues in parallel with increased MnSOD expression. Representative images of at least 15 different cases (Stage III, ductal invasive carcinoma) are shown.

glycolytic metabolism (Figs 2,3), drug resistance (Fig. 8) and enhanced capacity to grow in soft agar (Supplementary Fig. 12).

\section{Discussion}

Investigations dedicated to unravelling the role of MnSOD in tumorigenesis and cancer progression have yielded conflicting results in regard to the nature and potential effects of MnSOD in influencing cancer risk, prognosis, survival, susceptibility to therapy and likelihood of relapse. Recently, the consideration of MnSOD expression in specific contexts along with the functional status of other cellular antioxidant systems led to the more encompassing idea that MnSOD contributes to the progression of tumours towards aggressive phenotypes in which MnSOD enhances anti-apoptotic defenses and increases invasion ${ }^{47,48}$. The role of MnSOD as a primary participant and director of the malignant transformation process has been less appreciated; however, MnSOD and its product $\mathrm{H}_{2} \mathrm{O}_{2}$ have recently been shown to play critical roles in controlling OCRs and glucose uptake in mouse embryonic fibroblasts as these cells progress through the cell cycle from quiescence to proliferative states ${ }^{49}$ and in a variety of other systems where MnSOD and $\mathrm{H}_{2} \mathrm{O}_{2}$ affect survival and cellular function ${ }^{50-56}$. Our results provide direct evidence in support of the concept that MnSOD strongly suppresses mitochondrial oxidative metabolism while bolstering glycolysis by mechanisms that involve the rerouting of $\mathrm{O}_{2}$ towards the production of a steady flow of $\mathrm{mtH}_{2} \mathrm{O}_{2}$. MnSOD expression is regulated by inflammatory ${ }^{57,58}$, metabolic and stress-activated transcription factors ${ }^{59-61}$ and is generally expressed in response to an acute insult ${ }^{62,63}$. While the possibility that MnSOD provides protection against stress when acutely upregulated has been extensively scrutinized ${ }^{64-66}$, our results indicate that when persistently overexpressed at levels found in human cancers, MnSOD contributes to the progressive deterioration of mitochondrial bioenergetics and the activation of glycolysis via $\mathrm{mtH}_{2} \mathrm{O}_{2}$ production and AMPK activation. Interestingly, a similar role for SOD1, a cytosolic superoxide dismutase, has been described ${ }^{67}$. Although through a different mechanism (association with two casein kinase 1-gamma homologues), it was demonstrated that $\mathrm{H}_{2} \mathrm{O}_{2}$ generation was required to suppress mitochondrial respiration and stimulate glycolysis indicating, as proposed here, that the manipulation of intracellular levels of $\mathrm{H}_{2} \mathrm{O}_{2}$ may be of therapeutic utility in cancer. In fact, according to the results shown in Figs 1-5 and Supplementary Figs 1,2, the persistent upregulation of MnSOD with parallel increase in steady-state levels of $\mathrm{H}_{2} \mathrm{O}_{2}$ seem to be a distinctive feature of transformed cells that appears to satisfy critical energetic and metabolic necessities. Moreover, we found that repressing signals originating from MnSOD overexpression (such as $\mathrm{mtH}_{2} \mathrm{O}_{2}$, oxCaMKII and AMPK) strongly inhibit glycolysis without reactivating oxidative-based metabolism in mitochondria. Consequently, the interruption of 
$\mathrm{MnSOD} / \mathrm{mtH}_{2} \mathrm{O}_{2} / \mathrm{oxCaMKII} / \mathrm{AMPK}$ signalling results in the metabolic collapse of cells derived from advanced-stage cancers (MDA-MB-231 and transformed MCF10A-Er-Src) resulting in marked cell death. $\mathrm{mtH}_{2} \mathrm{O}_{2}$ was identified as the agent that integrates MnSOD-dependent mitochondrial signalling with the activation of AMPK, which was confirmed in experiments where we attenuated $\mathrm{mtH}_{2} \mathrm{O}_{2}$ levels by ectopically expressing mitochondria-targeted catalase, which rapidly dismutates $\mathrm{H}_{2} \mathrm{O}_{2}$ preventing its long-range effects. The effect of mitocatalase in repressing glycolysis in MnSOD-overexpressing cells indicates that there is an aggressive cancer-specific mechanism of glycolysis centred on the mitochondria-driven activation of the MnSOD/ AMPK axis. This conclusion is based on the observation that silencing MnSOD or inhibiting AMPK reduces glycolysis to baseline levels while only marginally affecting ATP levels in cells expressing low levels of MnSOD (MCF7/neo cells). Inhibition of glycolysis with 2-DG reduced steady-state ATP levels in all cell lines examined but only killed those expressing elevated MnSOD levels such as Mn44 and Mn11 cells. Taken together, with the observation that mitochondria-driven ATP production was severely impaired in Mn44 and Mn11 lines, it is proposed that MnSOD promotes survival independently of oxygen (a clinically relevant problem) at the expense of metabolic flexibility. In this regard, the identification of a specific mitochondria-toAMPK signalling axis that is activated by MnSOD upregulation and dependent on $\mathrm{mtH}_{2} \mathrm{O}_{2}$ in cancer cells can potentially be exploited for cancer therapeutic as this is not likely to interfere with normal glycolytic processes in healthy cells. This is especially important in view of recent findings demonstrating that AMPK activation is critical for the maintenance of cancer cell biosynthetic activity ${ }^{68}$.

On investigating the possible mechanisms by which AMPK is maintained constitutively activated in cells overexpressing MnSOD, we identified CaMKII as the redox-sensitive kinase activated upstream of AMPK, thereby translating the persistent increase in intracellular $\mathrm{H}_{2} \mathrm{O}_{2}$ into constitutive AMPK activation. The critical role of CaMKII in sustaining metabolic alterations required for cancer cell survival was illustrated by the effectiveness of KN93 in inhibiting AMPK phosphorylation and activity, and by its effectiveness in selectively killing MnSOD overexpressing cells as compared with neo control cells. Collectively, these experiments identified a mechanistic basis for the development of $\mathrm{KN} 93$ as an anticancer agent.

The likely clinical relevance of the data presented herein is further supported by the strong parallels between the results obtained in cell lines where MnSOD expression was manipulated and tissues obtained from patient biopsies where MnSOD, Bcl-2 and survivin were strongly upregulated in parallel with AMPK activation. The finding of elevated Bcl-2 and survivin expression in tumours has been generally associated with poor prognosis; therefore, mechanisms to suppress the expression of these antiapoptotic proteins in tumours are generally regarded as attractive therapeutic goals. By specifically targeting the $\mathrm{MnSOD} / \mathrm{mtH}_{2} \mathrm{O}_{2} /$ AMPK axis, treatments may be developed to limit the expression of Bcl-2 and survivin, thereby diminishing the reliance on existing therapies that eliminate cancer cells but have prominent detrimental off-target effects.

MnSOD upregulation, AMPK activation and a gradual shift towards glycolysis as described in this work are to be regarded as part of a multifaceted process that occur in carcinogenesis. We see the MnSOD/oxCaMKII/AMPK-dependent metabolic switch in cellular metabolism as a necessary occurrence for the successful establishment of malignancy that, based on the results presented herein, permits cells to survive oxygen deprivation while strongly promoting anti-apoptotic defenses. As alterations in cellular metabolism from mitochondria-based to glycolytic encompasses relevant to almost all malignant solid tumours, we think that these data identify the MnSOD/ $\mathrm{H}_{2} \mathrm{O}_{2} / \mathrm{AMPK}$ axis as a primary regulator of the Warburg effect and as such, a valuable new piece to the complex puzzle that is tumour progression.

\section{Methods}

Human patient sample analysis. De-identified human tissue samples were obtained from the University of Illinois at Chicago tissue bank according to IRB exemption note 20110082-58687-1/OPRS/UIC. Multiple images were acquired from at least six individual normal and six cancerous breast, prostate and colon tissues, as identified by Dr Andre Kajdacsy-Balla, a clinical pathologist. Representative images were used for Figs 1,4f,9 and Supplementary Fig. 1.

Fluorescent immunohistochemistry. Slides were blocked with regular fetal calf serum and incubated with primary antibody (MnSOD, LDH, diluted 1:1,000 in $1 \times$ TBST, Abcam, Cambridge, MA, USA), overnight at $4^{\circ} \mathrm{C}$. After rinsing in TBST, sections were incubated with Alexafluor-conjugated secondary antibodies (Invitrogen/Life Sciences, Grand Island, NY, USA). Slides were examined on a Nikon ECLIPSE E400 microscope and documented using the SPOT Advanced version 4.0.1 software.

TMA analysis. MnSOD expression in multiple molecular subtypes of breast cancer was assessed using immunofluorescent imaging of tissue microarray (TMA)-1005 (Protein Biotechnologies, Ramona, CA, USA). Protein was blocked with normal serum and incubated with anti-MnSOD, anti-pAMPK-Thr172 (diluted 1:100 in $1 \times$ TBST containing $0.1 \%$ bovine serum albumin, Abcam) overnight at $4{ }^{\circ} \mathrm{C}$. The slides were then incubated with Alexafluor-conjugated secondary antibodies (Invitrogen/Life Sciences) and examined on an Apotome (Zeiss, Jena, Germany). Relative fluorescent intensity was measured using ImageJ, and RFU values were associated with molecular subtypes described previously ${ }^{6}$.

Quantitation of relative fluorescent units. Corrected total fluorescence was calculated as described previously ${ }^{70}$. Three background samples were taken per selection to assure proper calibration.

Measurement of MnSOD activity. MnSOD was immunoprecipitated from cell lysates using a mouse anti-MnSOD antibody conjugated to protein A-bound resin. Protein $(20 \mu \mathrm{g})$ from lysates was loaded into PBS solutions containing $20 \mu \mathrm{g}$ antiMnSOD antibody. MnSOD activity was calculated compared with MCF7/neo based on the ability of MnSOD-bound to resin to inhibit the reduction of $50 \mu \mathrm{M}$ cytochrome $\mathrm{c} \mathrm{Fe}$ (III) reduced by hypoxanthine $(50 \mu \mathrm{M}) /$ xanthine oxidase

$\left(10 \mathrm{mU} \mathrm{ml}^{-1}\right)$ system. Incubations of MnSOD immobilized to resin, cytochrome $c$ and xanthine oxidase/hypoxanthine were vigorously agitated. Total cytochrome $c$ reduction was measured by absorbance at $550 \mathrm{~nm}$ after $30 \mathrm{~min}$ incubation at room temperature.

Confocal microscopy. Cells were plated onto MatTek glass-bottomed culture dishes and allowed to adhere overnight. After treatments, cells were washed with PBS and fixed with $4 \%$ paraformaldehyde (PFA). Cells were permeabilized using cold methanol. Images were recorded using a Zeiss LSM510UV META confocal microscope.

Amplex red assay. $\mathrm{H}_{2} \mathrm{O}_{2}$ production from cells was measured using the Amplex Red Hydrogen Peroxide/Peroxidase Assay Kit (Invitrogen/Life Sciences).

Mitocatalase transfection. Cells were grown in a six-well plate in media. Mitocatalase adenovirus was added to treatment wells and allowed to infect cells for $24 \mathrm{~h}$. Virus was a generous gift from Dr J. Andres Melendez (SUNY, Albany).

ATP assay. Cells were grown in a white-walled, clear bottom 96-well plate in media, transferred to glucose-free media with galactose and then analysed for ATP production using the Mitochondrial Tox-Glo Assay Kit (Promega, Madison, WI, USA) using a spectrophotometer.

JC-1 assay. Cells were grown in MatTek confocal dishes. On achieving $70 \%$ confluency, cells were incubated in $5 \mu \mathrm{M} \mathrm{JC}-1$ for $20 \mathrm{~min}$ at $37^{\circ} \mathrm{C}$ then washed $2 \times$ in PBS and immediately imaged by confocal microscopy.

Glycolysis assay. Cells were grown in a 96-well plate in media, transferred to serum-free media for $24 \mathrm{~h}$ and then analysed for glycolytic activity using the Glycolysis Cell-Based Assay Kit (Cayman Chemical, Ann Arbor, MI, USA). 
MnSOD/AMPK silencing. MnSOD, AMPK and scrambled siRNA were purchased from Santa Cruz Biotechnology (Santa Cruz, CA, USA) and incorporated into cells via electroporation using Amaxa Nucleofector Technology (Lonza, Basel, Switzerland). After electroporation, cells were plated and incubated for $24 \mathrm{~h}$, and then collected for protein analysis by western blot or plated for glycolysis measurements.

Flow cytometry. Cells were analysed using YOPRO and propidium iodide for apoptosis and viability by flow cytometry at the University of Illinois at Chicago Research Resources Flow Cytometry Service.

PFK, pyruvate kinase and hexokinase activity assays. Colorimetric assay kits were purchased from Abcam. Enzyme activities were measured following the manufacturer's instructions. In brief, equal amounts $\left(1-2 \times 10^{6}\right.$ cells $)$ of each cell line were lysed with cold assay buffer on ice, and the supernatant was mixed with freshly prepared reaction mix in 96-well plates. The plates were then incubated at $37^{\circ} \mathrm{C}$ for $60 \mathrm{~min}$, and absorbance was measured every $10 \mathrm{~min}$. Two time points $(20$ and $60 \mathrm{~min}$ ) were chosen to calculate the enzyme activity.

Transformation of MCF10A-Er-Src and silencing of MnSOD. For silencing of MnSOD in MCF10A(Er-Src) cells, Lipofectamine LTX (Invitrogen) was used as per the manufacturer's instructions. Silencing was performed using control scrRNA and MnSOD siRNA (Santa Cruz Biotechnologies). Briefly, cells were incubated with either scrRNA or siRNA at $37^{\circ} \mathrm{C}$ for $8 \mathrm{~h}$, and then treated with $1 \mu \mathrm{M}$ tamoxifen (Sigma Aldrich). The cells were harvested for analysis $48 \mathrm{~h}$ after tamoxifen was added. For transformation MCF10A-Er-Src cells were plated at 50\% confluency and maintained in $1 \mu \mathrm{M}$ tamoxifen for the duration of the experiment as indicated. MCF10AEr-Src cells were a kind gift from Dr Kevin Struhl (Harvard University)

Statistical analysis. Statistical analysis was performed with GraphPad InStat by using one-way analysis of variance with Student-Newman-Keuls comparison. A value of $P<0.05$ was considered significant, whereas values of $P<0.01$ and $P<0.001$ were considered highly significant.

\section{References}

1. Bell, E. L., Klimova, T. \& Chandel, N. S. Targeting the mitochondria for cancer therapy: regulation of hypoxia-inducible factor by mitochondria. Antioxid. Redox Signal. 10, 635-640 (2008).

2. Mullen, A. R. et al. Reductive carboxylation supports growth in tumour cells with defective mitochondria. Nature 481, 385-388 (2012)

3. Woo, D. K. et al. Mitochondrial genome instability and ROS enhance intestinal tumorigenesis in $\mathrm{APC}\left(\mathrm{Min}_{\mathrm{Min}} /+\right)$ mice. Am. J. Pathol. 180, 24-31 (2012).

4. Warburg, O. On respiratory impairment in cancer cells. Science 124, 269-270 (1956).

5. Tormos, K. V. et al. Mitochondrial complex III ROS regulate adipocyte differentiation. Cell Metab. 14, 537-544 (2011).

6. Rehman, J. et al. Inhibition of mitochondrial fission prevents cell cycle progression in lung cancer. FASEB J. 26, 2175-2186 (2012).

7. Majewski, N. et al. Hexokinase-mitochondria interaction mediated by Akt is required to inhibit apoptosis in the presence or absence of Bax and Bak. Mol. Cell 16, 819-830 (2004).

8. Brunelle, J. K. et al. c-Myc sensitization to oxygen deprivation-induced cell death is dependent on Bax/Bak, but is independent of p53 and hypoxiainducible factor-1. J. Biol. Chem. 279, 4305-4312 (2004).

9. Li, J. et al. PTEN, a putative protein tyrosine phosphatase gene mutated in human brain, breast, and prostate cancer. Science 275, 1943-1947 (1997).

10. Solomon, H., Madar, S. \& Rotter, V. Mutant p53 gain of function is interwoven into the hallmarks of cancer. J. Pathol. 225, 475-478 (2011).

11. Lim, Y. C. Y. et al. A role for homologous recombination and abnormal cell cycle progression in radioresistance of glioma initiating cells. Mol. Cancer Ther. 11, 1863-1872 (2012).

12. Kelly, P. N. \& Strasser, A. The role of Bcl-2 and its pro-survival relatives in tumourigenesis and cancer therapy. Cell Death Differ. 18, 1414-1424 (2011).

13. McDuff, F. K. \& Turner, S. D. Jailbreak: oncogene-induced senescence and its evasion. Cell Signal. 23, 6-13 (2011).

14. Bryson, J. M., Coy, P. E., Gottlob, K., Hay, N. \& Robey, R. B. Increased hexokinase activity, of either ectopic or endogenous origin, protects renal epithelial cells against acute oxidant-induced cell death. J. Biol. Chem. 277, 11392-11400 (2002).

15. Hamanaka, R. B. \& Chandel, N. S. Targeting glucose metabolism for cancer therapy. J. Exp. Med. 209, 211-215 (2012).

16. Wheaton, W. W. \& Chandel, N. S. Hypoxia. 2. Hypoxia regulates cellular metabolism. Am. J. Physiol. Cell Physiol. 300, C385-C393 (2011).

17. Glick, D. et al. BNip3 regulates mitochondrial function and lipid metabolism in the liver. Mol. Cell Biol. 32, 2570-2584 (2012).
18. Gordan, J. D., Thompson, C. B. \& Simon, M. C. HIF and c-Myc: sibling rivals for control of cancer cell metabolism and proliferation. Cancer Cell 12, 108-113 (2007).

19. Robey, R. B. \& Hay, N. Is Akt the "Warburg kinase"?-Akt-energy metabolism interactions and oncogenesis. Semin. Cancer Biol. 19, 25-31 (2009).

20. Marin-Valencia, I. et al. Analysis of tumor metabolism reveals mitochondrial glucose oxidation in genetically diverse human glioblastomas in the mouse brain in vivo. Cell Metab. 15, 827-837 (2012).

21. Ward, P. S. \& Thompson, C. B. Metabolic reprogramming: a cancer hallmark even warburg did not anticipate. Cancer Cell 21, 297-308 (2012).

22. Sun, Q. et al. Mammalian target of rapamycin up-regulation of pyruvate kinase isoenzyme type M2 is critical for aerobic glycolysis and tumor growth. Proc. Natl Acad. Sci. USA 108, 4129-4134 (2011).

23. Sgambato, A. et al. Cyclin E correlates with manganese superoxide dismutase expression and predicts survival in early breast cancer patients receiving adjuvant epirubicin-based chemotherapy. Cancer Sci. 100, 1026-1033 (2009).

24. Ennen, M. et al. Regulation of the high basal expression of the manganese superoxide dismutase gene in aggressive breast cancer cells. Free Radic. Biol. Med. 50, 1771-1779 (2011).

25. Quiros, I. et al. Upregulation of manganese superoxide dismutase (SOD2) is a common pathway for neuroendocrine differentiation in prostate cancer cells. Int. J. Cancer 125, 1497-1504 (2009).

26. Weaver, Z. A. et al. A recurring pattern of chromosomal aberrations in mammary gland tumors of MMTV-cmyc transgenic mice. Genes Chromosomes Cancer 25, 251-260 (1999).

27. Dhar, S. K., Tangpong, J., Chaiswing, L., Oberley, T. D. \& St Clair, D. K. Manganese superoxide dismutase is a p53-regulated gene that switches cancers between early and advanced stages. Cancer Res. 71, 6684-6695 (2011).

28. Ansenberger-Fricano, K. et al. The peroxidase activity of mitochondrial superoxide dismutase. Free Radic. Biol. Med. 54, 116-124 (2012).

29. Holley, A. K., Dhar, S. K. \& St Clair, D. K. Manganese superoxide dismutase versus p53: the mitochondrial center. Ann. N. Y. Acad. Sci. 1201, 72-78 (2010).

30. Holley, A. K., Dhar, S. K. \& St Clair, D. K. Manganese superoxide dismutase vs. p53: regulation of mitochondrial ROS. Mitochondrion 10, 649-661 (2010).

31. Kulawiec, M. et al. Tumorigenic transformation of human breast epithelial cells induced by mitochondrial DNA depletion. Cancer Biol. Ther. 7, 1732-1743 (2008).

32. Winder, W. W. \& Hardie, D. G. AMP-activated protein kinase, a metabolic master switch: possible roles in type 2 diabetes. Am. J. Physiol. 277, E1-10 (1999).

33. $\mathrm{Wu}, \mathrm{M}$. et al. Multiparameter metabolic analysis reveals a close link between attenuated mitochondrial bioenergetic function and enhanced glycolysis dependency in human tumor cells. Am. J. Physiol. Cell Physiol. 292, C125-C136 (2007).

34. Kao, K. J., Chang, K. M., Hsu, H. C. \& Huang, A. T. Correlation of microarraybased breast cancer molecular subtypes and clinical outcomes: implications for treatment optimization. BMC Cancer 11, 143 (2011).

35. Qing, G. et al. Combinatorial regulation of neuroblastoma tumor progression by N-Myc and hypoxia inducible factor HIF-1alpha. Cancer Res. 70, 10351-10361 (2010).

36. Dawson, D. M., Goodfriend, T. L. \& Kaplan, N. O. Lactic dehydrogenases: functions of the two types rates of synthesis of the two major forms can be correlated with metabolic differentiation. Science 143, 929-933 (1964).

37. Hirsch, H. A., Iliopoulos, D. \& Struhl, K. Metformin inhibits the inflammatory response associated with cellular transformation and cancer stem cell growth Proc. Natl Acad. Sci. USA 110, 972-977 (2013).

38. Iliopoulos, D., Hirsch, H. A., Wang, G. \& Struhl, K. Inducible formation of breast cancer stem cells and their dynamic equilibrium with non-stem cancer cells via IL6 secretion. Proc. Natl Acad. Sci. USA 108, 1397-1402 (2011).

39. Chaiswing, L., Zhong, W. \& Oberley, T. D. Increasing discordant antioxidant protein levels and enzymatic activities contribute to increasing redox imbalance observed during human prostate cancer progression. Free Radic. Biol. Med. 67, 342-352 (2014)

40. Zhang, M. et al. Thromboxane receptor activates the AMP-activated protein kinase in vascular smooth muscle cells via hydrogen peroxide. Circ. Res. 102, 328-337 (2008).

41. Jeon, S. M., Chandel, N. S. \& Hay, N. AMPK regulates NADPH homeostasis to promote tumour cell survival during energy stress. Nature 485, 661-665 (2012).

42. Marsin, A. S. et al. Phosphorylation and activation of heart PFK-2 by AMPK has a role in the stimulation of glycolysis during ischaemia. Curr. Biol. 10, $1247-1255$ (2000).

43. Erickson, J. R. et al. A dynamic pathway for calcium-independent activation of CaMKII by methionine oxidation. Cell 133, 462-474 (2008).

44. Luczak, E. D. \& Anderson, M. E. CaMKII oxidative activation and the pathogenesis of cardiac disease. J. Mol. Cell Cardiol. 73, 112-116 (2014).

45. Meng, Z. et al. Berbamine inhibits the growth of liver cancer cells and cancerinitiating cells by targeting $\mathrm{Ca}(2)(+) /$ calmodulin-dependent protein kinase II. Mol. Cancer Ther. 12, 2067-2077 (2013). 
46. Sullivan, L. B. \& Chandel, N. S. Mitochondrial metabolism in TCA cycle mutant cancer cells. Cell Cycle 13, 347-348 (2013).

47. Britschgi, A. et al. Calcium-activated chloride channel ANO1 promotes breast cancer progression by activating EGFR and CAMK signaling. Proc. Natl Acad. Sci. USA 110, E1026-E1034 (2013).

48. Knight, J. A. et al. Genetic variants of GPX1 and SOD2 and breast cancer risk at the Ontario site of the Breast Cancer Family Registry. Cancer Epidemiol. Biomarkers Prev. 13, 146-149 (2004).

49. Sarsour, E. H. et al. Manganese superoxide dismutase regulates a metabolic switch during the mammalian cell cycle. Cancer Res. 72, 3807-3816 (2012).

50. Cai, Y. et al. Increased oxygen radical formation and mitochondrial dysfunction mediate beta cell apoptosis under conditions of AMP-activated protein kinase stimulation. Free. Radic. Biol. Med. 42, 64-78 (2007).

51. Damelin, L. H. et al. Fat-loaded HepG2 spheroids exhibit enhanced protection from Pro-oxidant and cytokine induced damage. J. Cell Biochem. 101, 723-734 (2007).

52. Guo, H., Chen, Y., Liao, L. \& Wu, W. Resveratrol protects HUVECs from oxidized-LDL induced oxidative damage by autophagy upregulation via the AMPK/SIRT1 pathway. Cardiovasc. Drugs Ther. 27, 189-198 (2013).

53. Kang, M. C. et al. Dieckol isolated from brown seaweed Ecklonia cava attenuates type capital I, Ukrainiancapital I, Ukrainian diabetes in $\mathrm{db} / \mathrm{db}$ mouse model. Food Chem. Toxicol. 53, 294-298 (2013).

54. Marosi, K. et al. Long-term exercise treatment reduces oxidative stress in the hippocampus of aging rats. Neuroscience 226, 21-28 (2012).

55 . Zarse, K. et al. Impaired insulin/IGF1 signaling extends life span by promoting mitochondrial L-proline catabolism to induce a transient ROS signal. Cell Metab. 15, 451-465 (2012).

56. Chetboun, M. et al. Maintenance of redox state and pancreatic beta-cell function: role of leptin and adiponectin. J. Cell Biochem. 113, 1966-1976 (2012).

57. Bernard, D., Monte, D., Vandenbunder, B. \& Abbadie, C. The c-Rel transcription factor can both induce and inhibit apoptosis in the same cells via the upregulation of MnSOD. Oncogene 21, 4392-4402 (2002).

58. Saccani, S., Pantano, S. \& Natoli, G. Two waves of nuclear factor kappaB recruitment to target promoters. J. Exp. Med. 193, 1351-1359 (2001).

59. Vrailas-Mortimer, A. et al. A muscle-specific p38 MAPK/Mef2/MnSOD pathway regulates stress, motor function, and life span in Drosophila. Dev. Cell. 21, 783-795 (2011).

60. Na, H. K. et al. (-)-Epigallocatechin gallate induces Nrf2-mediated antioxidant enzyme expression via activation of PI3K and ERK in human mammary epithelial cells. Arch. Biochem. Biophys. 476, 171-177 (2008).

61. Drane, P., Bravard, A., Bouvard, V. \& May, E. Reciprocal down-regulation of p53 and SOD2 gene expression-implication in p53 mediated apoptosis. Oncogene 20, 430-439 (2001)

62. Negoro, S. et al. Activation of signal transducer and activator of transcription 3 protects cardiomyocytes from hypoxia/reoxygenation-induced oxidative stress through the upregulation of manganese superoxide dismutase. Circulation 104, 979-981 (2001).

63. Eldridge, A. et al. Manganese superoxide dismutase interacts with a large scale of cellular and mitochondrial proteins in low-dose radiation-induced adaptive radioprotection. Free Radic. Biol. Med. 53, 1838-1847 (2012).

64. Chen, C. H., Liu, K. \& Chan, J. Y. Anesthetic preconditioning confers acute cardioprotection via up-regulation of manganese superoxide dismutase and preservation of mitochondrial respiratory enzyme activity. Shock 29, 300-308 (2008).
65. Koch, O. R., De Leo, M. E., Borrello, S., Palombini, G. \& Galeotti, T. Ethanol treatment up-regulates the expression of mitochondrial manganese superoxide dismutase in rat liver. Biochem. Biophys. Res. Commun. 201, 1356-1365 (1994).

66. Lawler, J. M., Kwak, H. B., Kim, J. H. \& Suk, M. H. Exercise training inducibility of MnSOD protein expression and activity is retained while reducing prooxidant signaling in the heart of senescent rats. Am. J. Physiol. Regul. Integr. Comp. Physiol. 296, R1496-R1502 (2009).

67. Reddi, A. R. \& Culotta, V. C. SOD1 integrates signals from oxygen and glucose to repress respiration. Cell 152, 224-235 (2013).

68. Zhao, F. et al. Imatinib resistance associated with BCR-ABL upregulation is dependent on HIF-1alpha-induced metabolic reprograming. Oncogene 29, 2962-2972 (2010).

69. Carey, L. A. et al. Race, breast cancer subtypes, and survival in the Carolina Breast Cancer Study. JAMA 295, 2492-2502 (2006).

70. Burgess, A. et al. Loss of human Greatwall results in G2 arrest and multiple mitotic defects due to deregulation of the cyclin B-Cdc2/PP2A balance. Proc. Natl Acad. Sci. USA 107, 12564-12569 (2010).

\section{Acknowledgements}

We are indebted to Drs Larry W. Oberley (in memoriam) and Frederick Domann (University of Iowa) for the generous gift of cell lines stably overexpressing MnSOD Dr Kevin P. Claffey (University of Connecticut) for the generous gift of MCF-7-AMPK $\alpha 1$ - I - cells, Dr Kevin Struhl (Harvard University) for the gift of the MCF10A lines expressing v-Src under ER induction, Patricia Mavrogianis, Andrew Hall and Emily Ionetz at the Research Histology Core at the University of Illinois at Chicago for providing histology services, Dr Soumen Bera, Emmanuel Ansong, Paula Green, Alyssa Master and M. Saqib Baig for technical assistance, and for funding provided by the US Department of Defense (ARO no. 61758-LS) to M.G.B, the NCRR/NIH (S10RR027848) to M.G.B., 1R21CA182103-01 to A.M.D. and M.G.B. K.A.-F. and P.C.H. were supported by NIH T32 (HL072742). We also acknowledge gifts from RO1 CA101053 to A.M.D., NIH P01 HL60678 to R.D.M., CAPES-Coordenação de Aperfeiçoamento Pessoal, Ministério da Ciência e Tecnologia/Brazil-A109/2013 to M.G.B. and M.E.L.C.

\section{Author contributions}

The key experiments were performed by P.C.H., K.A.-F., M.M., A.L.A., D.N.E., D.G P.C.H., K.A.-F., M.M., A.L.A., D.N.E., A.M.D., J.H.S. and M.G.B. conceived and designed experiments. Data interpretation and hypothesis were generated by P.C.H., K.A.-F., M.M., A.M.D., A.K.-B., R.D.M., M.E.L.C., J.H.S., M.G.B. D.N.E., A.K.-B. and A.M.D. provided exclusive reagents and model systems. A.K.B. built, classified and analysed data from T.M.A. A.M.D., R.D.M., J.H.S., M.E.L.C. and M.G.B. wrote the manuscript. M.G.B. had the general oversight of the project.

\section{Additional information}

Supplementary Information accompanies this paper at http://www.nature.com/ naturecommunications

Competing financial interests: The authors declare no competing financial interests.

Reprints and permission information is available online at http://npg.nature.com/ reprintsandpermissions/

How to cite this article: Hart, P. C. et al. MnSOD upregulation sustains the Warburg effect via mitochondrial ROS and AMPK-dependent signalling in cancer. Nat. Commun 6:6053 doi: 10.1038/ncomms7053 (2015). 